\title{
Synthesis and Hypoglycemic and Anti-inflammatory Activity Screening of Novel Substituted 5-[Morpholino(Phenyl)Methyl]-Thiazolidine-2,4- Diones and Their Molecular Docking Studies
}

\author{
Yeni Sübstitüe 5-[Morfolino(Fenil)Metil]-Tiazolidin-2,4-Dionların Sentezi \\ ve Hipoglisemik ve Antienflamatuvar Aktivitelerinin Taranması ile \\ Moleküler Doking Çalışmaları
}

\author{
(D) Srikanth Kumar KARUMANCHI ${ }^{1}$, (D) Lakshmana Rao ATMAKURI ${ }^{1 *}$, (D) V Basaveswara Rao MANDAVA2 ${ }^{2}$ (D) Srikala RAJALA 3 \\ 1V. V. Institute of Pharmaceutical Sciences, Department of Pharmaceutical Chemistry, Gudlavalleru, Andhra Pradesh, India \\ 2Krishna University, Department of Chemistry, Machilipatnam, Andhra Pradesh, India \\ 3Sree Vidyanikethan College of Pharmacy, Department of Pharmaceutical Chemistry, Tirupati, Andhra Pradesh, India
}

\begin{abstract}
Objectives: The aim was the synthesis of novel substituted 5-[morpholino(phenyl)methyl]-thiazolidine-2,4-diones and screening for their in vivo hypoglycemic activity and in vitro anti-inflammatory activity, as well as molecular docking studies to find out active potential lead molecules.

Materials and Methods: Substituted aromatic aldehydes, thiazolidine-2,4-dione, and morpholine on Mannich reaction gave the title compounds. They were characterized by physical and spectral methods. In vivo hypoglycemic activity was examined in alloxan induced Wistar albino rats by tail tipping method. In vitro anti-inflammatory activity was tested by human red blood cell (HRBC) membrane stabilization and protein denaturation. Using AutoDock, molecular docking studies were carried out to find out the best fit ligands.

Results: Series of substituted 5-[morpholino(phenyl)methyl]-thiazolidine-2,4-diones were synthesized and chemically they were confirmed by spectral techniques. Acute toxic studies of in vivo hypoglycemic activity results revealed that compounds $4 \mathrm{c}$, $4 \mathrm{~h}$, and $4 \mathrm{n}$ exhibited good activity at $35 \mathrm{mg} / \mathrm{kg}$ body weight. Chronic toxic study results indicated that compounds $4 \mathrm{~h}$ and $4 \mathrm{n}$ exhibited good activity at $70 \mathrm{mg} / \mathrm{kg}$ body weight. Antiinflammatory activity results indicated the highest inhibition was shown by compounds $4 \mathrm{k}$ and $4 \mathrm{f}$ at $500 \mu \mathrm{g} / \mathrm{mL}$ in $\mathrm{HRBC} \mathrm{membrane}$ stabilization. In protein denaturation, the highest inhibition was shown by compound $4 \mathrm{k}$ at $500 \mu \mathrm{g} / \mathrm{mL}$. In molecular docking studies, compounds $4 \mathrm{~h}$ and $4 \mathrm{n}$ exhibited higher binding affinity at PPAR $\gamma$ receptor protein and compound 4k exhibited higher binding affinity at COX-1 and COX-2 actives sites.

Conclusion: Microwave irradiation produced high yield in short reaction times. The presence of electron releasing groups at the para position of the phenyl ring may give the ability to produce hypoglycemic activity and the presence of electron withdrawing groups at the para position of the phenyl ring causes anti-inflammatory activity. The results showed that some compounds exhibited good hypoglycemic and anti-inflammatory activities. Compounds $4 \mathrm{~h}$ and $4 \mathrm{n}$ exhibited higher binding affinity at PPAR $\gamma$ receptor protein and compound $4 \mathrm{k}$ exhibited higher binding affinity at COX isoenzymes' active sites in molecular docking studies.
\end{abstract}

Key words: Thiazolidinediones bearing morpholine, Mannich reaction, in vivo hypoglycemic activity, in vitro anti-inflammatory activity, docking studies

öz

Amaç: Bu çalışmanın amacı, yeni sübstitüe 5-[morfolino(fenil)metil]-tiyazolidin-2,4-dionların sentezi ve in vivo hipoglisemik ve in vitro antienflamatuvar aktivitelerinin taranması ile olası aktif moleküller için moleküler doking çalıșmalarının yapılmasıdır.

Gereç ve Yöntemler: Bileșikler; sübstitüe aromatik aldehidler, tiyazolidin-2,4-dion ve morfolinin mannich reaksiyonu sonucu elde edilmiş, elde edilen bileşikler fiziksel ve spektral yöntemlerle karakterize edilmiștir. In vivo hipoglisemik aktivite, alloxan ile indüklenen Wistar albino farelerde "tail

*Correspondence: E-mail: dralrao@gmail.com, Phone: +09848779133 ORCID: orcid.org/0000-0001-5601-037X

Received: 04.05.2018, Accepted: 20.07.2018

๑Turk J Pharm Sci, Published by Galenos Publishing House. 
tipping" yöntemi, in vitro anti-enflamatuar aktivite ise, insan kırmızı kan hücrelerinde membran stabilizasyonu ve protein denatürasyon yöntemleri ile gerçekleştirilmiştir. AutoDock kullanarak, en uygun ligandları bulmak için moleküler doking çalışmaları yapılmıştır.

Bulgular: Sübstitüe 5-[morfolino(fenil)metil]-tiyazolidin-2,4-dion serileri sentezlenmiș ve spektral tekniklerle kimyasal yapıları teyit edilmiştir. In vivo hipoglisemik aktivite sonuçlarıyla ilgili akut toksik çalıșmaları, 4c, 4h ve $4 \mathrm{n}$ bileșiklerinin $35 \mathrm{mg} / \mathrm{kg}$ 'da, kronik toksisite çalışmaları da, bileşik $4 \mathrm{~h}$ ve $4 n^{\prime}$ in $70 \mathrm{mg} / \mathrm{kg}$ 'da iyi aktivite sergilediklerini göstermektedir. Antienflamatuvar aktivite sonuçları, membran stabilizasyon yönteminde en yüksek

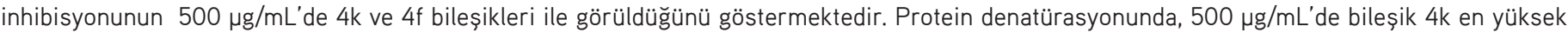
inhibisyonu göstermiştir. Moleküler doking çalıșmalarında, 4h ve 4n bileşiklerinin PPAR $\gamma$ reseptör proteinine, 4k bileşiğinin de, COX-1 ve COX-2 aktif bölgelerine daha yüksek bağlanma afinitesi sergilediği görülmüștür.

Sonuç: Mikrodalga tekniği düşük reaksiyon sürelerinde yüksek verim sağlamıștır. Fenil halkasının para pozisyonunda; elektron salıcı grupların hipoglisemik aktivite oluşturabileceği, elektron çekici grupların ise antienflamatuvar aktiviteye neden olabileceği belirtilmiştir. Sonuçla, bazı bileşiklerin iyi hipoglisemik ve anti-enflamatuar aktivite sergilediklerini göstermiștir.

Moleküler doking çalışmalarında; bileșik $4 \mathrm{~h}$ ve $4 \mathrm{n}$, PPAR $\gamma$ reseptör proteininde, bileșik 4k da COX izoenzimleri aktif bölgelerinde daha yüksek bağlanma afinitesi sergilemiştir.

Anahtar kelimeler: Morfolin taşıyan tiyazolidinonlar, mannich reaksiyonu, in vivo hipoglisemik aktivite, in vitro antienflamatuvar aktivite, doking çalıșmaları

\section{INTRODUCTION}

Diabetes mellitus (DM) is a common chronic metabolic syndrome in which high blood sugar levels occur over a prolonged time and symptoms include frequent urination, increased hunger, and increased thirst. DM is associated with severe degenerative complications such as nephropathy, neuropathy, cataract, retinopathy, accelerated atherosclerosis, stroke, and increased risk of myocardial infarction. Onset of these pathologies is a remarkable event in the course of both type 1 and type 2 diabetes. Prevention and control are still serious challenging therapeutic problems as they stand for the foremost causes of morbidity and mortality for diabetic patients. Type 1 diabetes is characterized by deficient insulin production and symptoms include excessive thirst (polydipsia) and excretion of urine (polyuria), weight loss, constant hunger, fatigue, and vision changes requiring daily administration of insulin. Type 2 diabetes results from the body's ineffective impaired insulin action. The majority of cases around the world are type 2 diabetes and it is principally the result of physical inactivity and excess body weight. Symptoms are similar to type 1 diabetes, but are less marked. In the past this type of diabetes was seen only in the adults but at present it is also happening frequently even in children. Normally in humans up to $80 \%$ of insulin stimulated glucose disposal takes place in the skeletal muscle, which is the major site of insulin resistance in type 2 diabetes. $^{2}$

Worldwide the frequency of diabetes for all age groups was estimated to be $2.8 \%$ in 2000 and $4.4 \%$ in 2030 . The total number of people with diabetes is expected to increase from 171 million in 2000 to 366 million in 2030. The occurrence of diabetes is higher in men than in women; however, there are more women with this diabetes than men. In developing countries, the urban population is expected to double between 2000 and 2030 . Across the world the most important demographic change to diabetes prevalence appears to be an increase in the proportion of people greater than 65 years of age. Therefore, there is a need to develop novel drug candidates in this region. ${ }^{3}$

Heterocyclic compounds and their derivatives are a fascinating field in medicinal chemistry because of their remarkable biological and pharmacological properties. In the family of heterocyclic compounds, heterocycles with $\mathrm{O}, \mathrm{S}$, and $\mathrm{N}$ atoms have attracted more attention in chemistry research due to their wide range of biological activities. $\mathrm{O}$ and $\mathrm{N}$ atoms containing morpholine have a unique position in heterocyclic chemistry. Morpholine is a six-member saturated heterocyclic system having $\mathrm{O}$ and $\mathrm{N}$ as heteroatoms at the $1^{\text {st }}$ and $4^{\text {th }}$ positions, respectively. Clinically the morpholine ring contains drugs used in the market that have considerable importance, such as timolol ( $\beta$-adrenergic blocker used in ocular hypertension and glaucoma), moclobemide (mono amino oxidase inhibitor used to treat depression and anxiety), reboxetine (norepinephrine reuptake inhibitor used as an antidepressant), pholcodine (opioid cough suppressant), emorfazone (nonsteroidal antiinflammatory agent), gefitinib (epidermal growth factor receptor inhibitor used for certain breast, lung, and other cancers), and linezolid (antibiotic used to treat infections caused by grampositive bacteria). ${ }^{4}$

Similarly, the thiazolidine-2,4-dione (TZD) nucleus is also one of the most important heterocyclics that has received much attention especially in the treatment of diabetes. TZD is a fivemember heterocyclic compound that consists of $S$ and $N$ as heteroatoms in the ring at the $1^{\text {st }}$ and $3^{\text {rd }}$ positions, respectively, along with ketone functional groups at the $2^{\text {nd }}$ and $4^{\text {th }}$ positions. The TZD class of drugs includes troglitazone, ciglitazone, rosiglitazone, and ciglitazone with an insulin sensitizing property. Troglitazone was withdrawn from the market in 2000 due to its hepatotoxicity producing nature. TZDs are found to produce hypoglycemic activity by lowering the blood glucose levels significantly through the activation of peroxisome proliferator-activated receptor gamma (PPAR $\gamma$ ) receptors. ${ }^{5}$ TZDs enhance the insulin action and promote glucose utilization in the peripheral tissues. The exact mechanism of TZDs has not been elucidated, but is expected to exhibit pharmacological actions by binding and agonistic properties at the PPAR $\gamma$ nuclear receptor. 6

Recently TZDs have been found to possess a wide range of biological activities such as antidiabetic, ${ }^{7}$ protein tyrosine phosphatise 1B inhibitory, ${ }^{8}$ 15-hydroxyprostaglandin 
dehydrogenase (15-PGDH) inhibitory, ${ }^{9}$ hypolipidemic, ${ }^{10}$ aldose reductase inhibitory, ${ }^{11}$ anti-inflammatory, ${ }^{12}$ antimicrobial, ${ }^{13}$ antitubercular, ${ }^{14}$ antioxidant, ${ }^{15}$ antitumor, ${ }^{16}$ and antiproliferative. ${ }^{17}$ From the literature observations, we made an attempt to design and develop novel TZD derivatives bearing morpholine in their structures using the Mannich reaction by both conventional heating and microwave irradiated methods. Microwave techniques give high yields, decrease by-product formation, and decrease the decomposition of products as compared to the conventional heating reaction methods.18,19 The developed compounds were characterized and screened for biological activities. By using AutoDock molecular docking software the binding energies of the designed ligands and their interactions at the target protein were studied.

\section{MATERIALS AND METHODS}

All the chemicals required for the synthesis of novel thiazolidinediones such as reagents and solvents were obtained from commercial suppliers in Merck grade and further they were used without purification. Reaction progress and completion were monitored by thin layer chromatography with the help of $0.25 \mathrm{~mm}$ E. Merck grade silica gel 60GF-254 precoated TLC plates; spots were observed under ultraviolet (UV)-light and in an iodine chamber. Fourier Transform Infrared Spectrometer (FT-IR) spectra of the compounds were recorded with a Bruker FT-IR analyzer spectrophotometer by compression of compound with anhydrous $\mathrm{KBr}$ under vacuum using the $\mathrm{KBr}$ pressed pellet technique. Chemical shifts in $\delta$, ppm of proton nuclear magnetic resonance ('H-NMR) and carbon-13 nuclear magnetic resonance $\left({ }^{13} \mathrm{C}-\mathrm{NMR}\right)$ spectra were recorded on a Bruker AMX $400 \mathrm{MHz}$ spectrometer using deuterated dimethyl sulfoxide (DMSO) solvent and tetramethylsilane as internal standard. Mass spectra of the compounds were recorded on an Agilent LC-MSD 1200 mass spectrometer. For conventional synthesis normal reflux condenser setup and microwave assisted synthesis was performed on an RGSSIRR model Raga's scientific microwave system having different power levels from $140 \mathrm{~W}$ to $700 \mathrm{~W}$. Melting points were determined by electrical melting point apparatus and were uncorrected. The Wistar albino rats used in the hypoglycemic study were divided into various groups and each group contained 6 rats $(n=6)$.

\section{Chemistry}

Synthesis of thiazolidine-2,4-dione (3)

A. Conventional synthesis: Chloroacetic acid $(20 \mathrm{mmoL})$ and thiourea (20 mmoL) were separately dissolved in $5 \mathrm{~mL}$ of water. The contents of the vessels were transferred into a three-necked round bottom flask and stirred until white precipitate was obtained. The reaction mixture was cooled and conc. Hydrochloric acid $[(\mathrm{HCl}) 6 \mathrm{~mL}]$ was added slowly to it from the dropping funnel. It was refluxed by applying gentle heat for about $10-12 \mathrm{~h}$ at $100-110^{\circ} \mathrm{C}$. The contents of the flask were cooled to solidify them and they were filtered to obtain the product by washing with water. Recrystallization was done using ethyl alcohol. ${ }^{20}$
B. Microwave irradiation synthesis: A mixture of chloroacetic acid $(10 \mathrm{mmoL})$ and thiourea $(10 \mathrm{mmoL})$ dissolved in $5 \mathrm{~mL}$ of water was transferred into the microwave synthesizer reaction vessel. The reaction vessel was closed with the help of lids and condenser and stirred for about $1 \mathrm{~h}$ in cold condition. Then $3 \mathrm{~mL}$ of conc. $\mathrm{HCl}$ was added to the reaction mixture and irradiated for 6 min using $280 \mathrm{~W}$ power level at $120^{\circ} \mathrm{C}$. The reaction mixture was cooled to room temperature and the obtained solid was filtered, dried, and recrystallized from ethyl alcohol. ${ }^{21}$

$78.42 \%$ (conventional synthesis yield), 90.25\% (MWI synthesis yield), white crystalline powder, melting point $124-126^{\circ} \mathrm{C}, \mathrm{R}_{\mathrm{f}}$ value 0.62 from using $9: 1 \mathrm{v} / \mathrm{v}$ of chloroform and methanol. IR [KBr v cm${ }^{-1}$ : $3321.46(-\mathrm{NH}-), 1689.94(\mathrm{C}=0), 2968.89$ (CH), 1303.29 (C-N), 626.69 (C-S). 'H-NMR [400 MHz, $\delta, p p m$, DMSO- $d_{6}$ ]: 12.015 (s, 1H, NH), 4.132 (s, 2H, CH ${ }_{2}$ ). ${ }^{13} \mathrm{C}-\mathrm{NMR}[400$ $\left.\mathrm{MHz}, \delta, \mathrm{ppm}, \mathrm{DMSO}-d_{6}\right]: 35.80,173.06,173.82$. ESI-MS: $\left(\mathrm{M}^{+}\right) \mathrm{m} / \mathrm{z}$ 117.

\section{General procedure for synthesis of compounds $4 a-4 n$}

A. Conventional synthesis: $0.01 \mathrm{~mol}$ of TZD (3) was dissolved in $5 \mathrm{~mL}$ of ethanol and $0.01 \mathrm{moL}$ of substituted aromatic benzaldehyde was added to this solution. The mixture was stirred for about $30 \mathrm{~min}$ at room temperature. To the reaction mixture $0.01 \mathrm{moL}$ of morpholine and a catalytic amount of conc. $\mathrm{HCl}$ (3-5 drops) were added, followed by refluxing for 5-6 $\mathrm{h}$. Completion of the reaction was monitored by TLC using mobile phase $\mathrm{n}$-hexane and ethylacetate (9:1). The reaction mixture was allowed to cool for about 2-4 $\mathrm{h}$ and then it was poured into ice cold water. The product was collected by filtration and washed with cold water followed by dry toluene. It was dried and recrystallized with absolute ethanol. ${ }^{22}$

B. Microwave irradiation synthesis: $0.01 \mathrm{moL}$ of TZD (3) was dissolved in $5 \mathrm{~mL}$ of ethanol and $0.01 \mathrm{moL}$ of substituted aromatic benzaldehyde was added to it. The reaction mixture was stirred for $30 \mathrm{~min}$ at RT. To the above solution $0.01 \mathrm{moL}$ of morpholine and 3-5 drops of conc. $\mathrm{HCl}$ were added. It was mixed well and placed in a Raga's scientific microwave synthesizer vessel and the reaction mixture was irradiated at $420 \mathrm{~W}$ power level for about $6-10 \mathrm{~min}$ at $120^{\circ} \mathrm{C}$. The reaction mixture was cooled to room temperature and treated with ice cold water for the work up process. The obtained precipitate was filtered, washed, and dried to get the desired compound. Completion of the reaction was monitored by TLC using the same mobile phase mentioned in the above conventional synthesis. ${ }^{23}$

5-[(4-chlorophenyl)-morpholin-4-yl-methyl]-thiazolidine-2,4dione (4a)

IR [KBr r cm$\left.{ }^{-1}\right]: 3331.29(-\mathrm{NH}-), 1660.56(\mathrm{C}=0), 1263.44(\mathrm{C}-\mathrm{N})$, $2984.21(\mathrm{C}-\mathrm{H}), 3045.20$ (=C-H), 615.50 (C-S), 1104.13 (C-O-C), 785.56 (C-Cl). ${ }^{1} \mathrm{H}-\mathrm{NMR}$ [400 MHz, $\delta, \mathrm{ppm}, \mathrm{DMSO}-\mathrm{d}_{6}$ ]: 12.105 (s, $1 \mathrm{H}, \mathrm{NH}$ ), 4.305-4.317 (d, 1H, TZD CH), 4.012-4.041 (d, 1H, N-CH), 7.355-7.421 (d, 2H, 3'-H\&5'-H), 6.945-7.014 (d, 2H, 2'-H\&6'-H), 3.642-3.685 (t, 4H, $\left.\mathrm{CH}_{2}-\mathrm{O}-\mathrm{CH}_{2}\right), 2.475-2.635\left(\mathrm{t}, 4 \mathrm{H}, \mathrm{CH}_{2}-\mathrm{N}-\mathrm{CH}_{2}\right.$ ). ${ }^{13} \mathrm{C}-\mathrm{NMR}\left[400 \mathrm{MHz}, \delta, \mathrm{ppm}, \mathrm{DMSO}-d_{6}\right.$ ]: $173.15,166.56,138.45$, 133.74, 130.32, 126.46, 68.44, 59.20, 56.48, 51.52. ESI-MS: $\left(M^{+}\right)$ $\mathrm{m} / \mathrm{z} 326$. 
5-[(2,4-dichlorophenyl)-morpholin-4-yl-methyl]-thiazolidine2,4-dione (4b)

IR [KBr r cm$\left.{ }^{-1}\right]: 3456.54(-\mathrm{NH}-), 1672.55(\mathrm{C}=\mathrm{O}), 1284.56(\mathrm{C}-\mathrm{N})$, $2949.85(\mathrm{C}-\mathrm{H}), 3084.78(=\mathrm{C}-\mathrm{H}), 632.04$ (C-S), 1131.45 (C-O-C), $796.74(\mathrm{C}-\mathrm{Cl}) .{ }^{1} \mathrm{H}-\mathrm{NMR}$ [400 MHz, $\left.\delta, \mathrm{ppm}, \mathrm{DMSO}-d_{6}\right]: 12.214$ (s, $1 \mathrm{H}, \mathrm{NH}), 4.321-4.348(\mathrm{~d}, 1 \mathrm{H}, \mathrm{TZD} \mathrm{CH}), 4.124-4.168(\mathrm{~d}, 1 \mathrm{H}, \mathrm{N}-\mathrm{CH})$, $7.409\left(\mathrm{~s}, 1 \mathrm{H}, 3^{\prime}-\mathrm{H}\right), 7.358-7.414\left(\mathrm{~d}, 1 \mathrm{H}, 5^{\prime}-\mathrm{H}\right), 6.984-7.021(\mathrm{~d}, 1 \mathrm{H}$, $\left.6^{\prime}-\mathrm{H}\right), 3.748-3.894\left(\mathrm{t}, 4 \mathrm{H}, \mathrm{CH}_{2}-\mathrm{O}-\mathrm{CH}_{2}\right), 2.546-2.798\left(\mathrm{t}, 4 \mathrm{H}, \mathrm{CH}_{2}-\right.$ $\mathrm{N}-\mathrm{CH}_{2}$ ). ${ }^{13} \mathrm{C}-\mathrm{NMR}\left[400 \mathrm{MHz}, \delta, \mathrm{ppm}, \mathrm{DMSO}-d_{6}\right.$ ]: 176.15, 167.08, 140.84, 136.56, 133.45, 131.63, 128.23, 125.46, 69.52, 60.45, 54.62, 50.45. ESI-MS: $\left(\mathrm{M}^{+}\right) \mathrm{m} / \mathrm{z} 361$.

5-[(4-fluorophenyl)-morpholin-4-yl-methyl]-thiazolidine-2,4dione (4c)

IR [KBr $\left.\vee \mathrm{cm}^{-1}\right]: 3425.45(-\mathrm{NH}-), 1673.52(\mathrm{C}=\mathrm{O}), 1274.25(\mathrm{C}-\mathrm{N})$, $2983.44(\mathrm{C}-\mathrm{H}), 3063.74(=\mathrm{C}-\mathrm{H}), 623.24$ (C-S), 1121.47 (C-O-C), 1345.25 (C-F). ${ }^{1} \mathrm{H}-\mathrm{NMR}\left[400 \mathrm{MHz}, \delta, \mathrm{ppm}, \mathrm{DMSO}-d_{6}\right.$ ]: 12.046 (s, $1 \mathrm{H}, \mathrm{NH}), 4.215-4.298(\mathrm{~d}, 1 \mathrm{H}, \mathrm{TZD} \mathrm{CH}), 4.112-4.156(\mathrm{~d}, 1 \mathrm{H}, \mathrm{N}-\mathrm{CH})$, 7.284-7.301 (d, 2H, 3'-H\&5'-H), 6.845-7.001 (d, 2H, 2'-H\&6'-H), 3.542-3.599 (t, 4H, $\mathrm{CH}_{2}-\mathrm{O}-\mathrm{CH}_{2}$ ), 2.546-2.741 (t, 4H, $\mathrm{CH}_{2}-\mathrm{N}-\mathrm{CH}_{2}$ ). ${ }^{13} \mathrm{C}-\mathrm{NMR}\left[400 \mathrm{MHz}, \delta, \mathrm{ppm}, \mathrm{DMSO}-d_{6}\right.$ ]: 175.26, 168.54, 139.74, $134.52,130.66,124.48,67.47,58.45,54.74,50.62$. ESI-MS: $\left(M^{+}\right)$ $\mathrm{m} / \mathrm{z} 310$.

5-[(4-hydroxyphenyl)-morpholin-4-yl-methyl]-thiazolidine-2,4dione (4d)

IR [KBr $\left.\vee \mathrm{cm}^{-1}\right]: 3415.41(-\mathrm{NH}-), 1681.46(\mathrm{C}=\mathrm{O}), 1264.74(\mathrm{C}-\mathrm{N})$, $2986.21(\mathrm{C}-\mathrm{H}), 3085.46$ (=C-H), 631.42 (C-S), 1116.22 (C-O-C), $3505.46(\mathrm{Ph}-\mathrm{OH}) .{ }^{1} \mathrm{H}-\mathrm{NMR}$ [400 MHz, $\delta, \mathrm{ppm}, \mathrm{DMSO}-d_{6}$ ]: 11.965 (s, 1H, NH), 4.317-4.365 (d, 1H, TZD CH), 9.845 (s, 1H, $\mathrm{C}_{6} \mathrm{H}_{4}-$ $\mathrm{OH}), 4.205-4.246(\mathrm{~d}, 1 \mathrm{H}, \mathrm{N}-\mathrm{CH}), 6.935-7.459\left(\mathrm{~m}, 4 \mathrm{H}, \mathrm{C}_{6} \mathrm{H}_{4}-\mathrm{OH}\right)$, 3.645-3.726 (t, 4H, $\mathrm{CH}_{2}-\mathrm{O}-\mathrm{CH}_{2}$ ), 2.685-2.784 (t, 4H, $\mathrm{CH}_{2}-\mathrm{N}-\mathrm{CH}_{2}$ ). ${ }^{13} \mathrm{C}-\mathrm{NMR}\left[400 \mathrm{MHz}, \delta, \mathrm{ppm}, \mathrm{DMSO}-d_{6}\right]: 176.46,167.89,159.56$, 138.46, 133.47, 115.48, 65.32, 59.64, 53.12, 51.32. ESI-MS: $\left(M^{+}\right)$ $\mathrm{m} / \mathrm{z} 308$.

5-[(4-methylphenyl)-morpholin-4-yl-methyl]-thiazolidine-2,4dione (4e)

IR [KBr r cm$\left.{ }^{-1}\right]: 3335.66(-\mathrm{NH}-), 1666.22(\mathrm{C}=\mathrm{O}), 1272.65(\mathrm{C}-\mathrm{N})$, 2980.67 (C-H), 3095.26 (=C-H), 615.22 (C-S), 1128.27 (C-O-C). ${ }^{1} \mathrm{H}-\mathrm{NMR}\left[400 \mathrm{MHz}, \delta, \mathrm{ppm}, \mathrm{DMSO}-d_{6}\right.$ ]: 12.025 (s, $\left.1 \mathrm{H}, \mathrm{NH}\right), 4.225-$ 4.308 (d, 1H, TZD CH), 2.865 (s, 3H, $\mathrm{C}_{6} \mathrm{H}_{4}-\mathrm{CH}_{3}$ ), 4.056-4.116 (d, $1 \mathrm{H}, \mathrm{N}-\mathrm{CH}), 6.849-7.554\left(\mathrm{~m}, 4 \mathrm{H}, \mathrm{C}_{6} \underline{\mathrm{H}}_{4}-\mathrm{CH}_{3}\right), 3.720-3.842(\mathrm{t}, 4 \mathrm{H}$, $\mathrm{CH}_{2}-\mathrm{O}-\mathrm{CH}_{2}$ ), 2.784-2.951 (t, 4H, $\left.\mathrm{CH}_{2}-\mathrm{N}-\mathrm{CH}_{2}\right) .{ }^{13} \mathrm{C}-\mathrm{NMR}[400$ $\mathrm{MHz}, \delta, \mathrm{ppm}$, DMSO-d ] : 178.66, 165.42, 158.45, 137.45, 134.62, 118.61, 66.30, 60.52, 54.11, 52.55, 30.28. ESI-MS: $\left(M^{+}\right)$m/z 306.

5-[(4-nitrophenyl)-morpholin-4-yl-methyl]-thiazolidine-2,4dione (4f)

IR $\left[\mathrm{KBr} \mathrm{r} \mathrm{cm}{ }^{-1}\right]: 3364.22(-\mathrm{NH}-), 1693.45(\mathrm{C}=\mathrm{O}), 1274.66(\mathrm{C}-\mathrm{N})$, $2968.41(\mathrm{C}-\mathrm{H}), 3095.45(=\mathrm{C}-\mathrm{H}), 618.62$ (C-S), $1135.84(\mathrm{C}-\mathrm{O}-\mathrm{C})$, 1362.04, $1546.15\left(\mathrm{NO}_{2}\right) .{ }^{1} \mathrm{H}-\mathrm{NMR}\left[400 \mathrm{MHz}, \delta, \mathrm{ppm}, \mathrm{DMSO}-d_{6}\right.$ ]: $12.546(\mathrm{~s}, 1 \mathrm{H}, \mathrm{NH}), 4.015-4.068(\mathrm{~d}, 1 \mathrm{H}, \mathrm{TZD} \mathrm{CH}), 4.325-4.366$ (d, $1 \mathrm{H}, \mathrm{N}-\mathrm{CH}), 6.846-7.387\left(\mathrm{~m}, 4 \mathrm{H}, \mathrm{C}_{6} \mathrm{H}_{4}-\mathrm{NO}_{2}\right), 3.862-3.984$ (t, $\left.4 \mathrm{H}, \mathrm{CH}_{2}-\mathrm{O}-\mathrm{CH}_{2}\right), 2.855-2.948\left(\mathrm{t}, 4 \mathrm{H}, \mathrm{CH}_{2}-\mathrm{N}-\mathrm{CH}_{2}\right) .{ }^{13} \mathrm{C}-\mathrm{NMR}[400$ $\mathrm{MHz}, \delta, \mathrm{ppm}, \mathrm{DMSO}-d_{6}$ ]: 178.79, 168.46, 150.62, 143.52, 130.46, 120.84, 68.32, 59.23, 55.74, 50.85. ESI-MS: $\left(M^{+}\right)$m/z 337. 5-[(4-methoxyphenyl)-morpholin-4-yl-methyl]-thiazolidine2,4-dione (4g)

IR [KBr r cm$\left.{ }^{-1}\right]: 3381.47(-\mathrm{NH}-), 1679.22(\mathrm{C}=\mathrm{O}), 1269.61(\mathrm{C}-\mathrm{N})$, $2971.52(\mathrm{C}-\mathrm{H}), 3068.94(=\mathrm{C}-\mathrm{H}), 629.41$ (C-S), 1130.49 (C-O-C). ${ }^{1} \mathrm{H}-\mathrm{NMR}\left[400 \mathrm{MHz}, \delta, \mathrm{ppm}, \mathrm{DMSO}-d_{6}\right.$ ]: 11.985 (s, 1H, NH), 4.2114.285 (d, 1H, TZD CH), 4.389-4.401 (d, 1H, N-CH), 6.956-7.421 (m, 4H, $\left.\mathrm{C}_{6} \underline{\mathrm{H}}_{4}-\mathrm{OCH}_{3}\right), 3.986\left(\mathrm{~s}, 3 \mathrm{H}, 4^{\prime}-\mathrm{OCH}_{3}\right), 3.745-3.889(\mathrm{t}, 4 \mathrm{H}$, $\left.\mathrm{CH}_{2}-\mathrm{O}-\mathrm{CH}_{2}\right), 2.651-2.894\left(\mathrm{t}, 4 \mathrm{H}, \mathrm{CH}_{2}-\mathrm{N}-\mathrm{CH}_{2}\right) \cdot{ }^{13} \mathrm{C}-\mathrm{NMR}[400$ $\mathrm{MHz}, \delta, \mathrm{ppm}$, DMSO-d ]: 180.22, 169.56, 160.55, 133.52, 129.44, 118.22, 68.45, 64.21, 59.45, 54.66, 51.23. ESI-MS: (M+) m/z 322. 5-[(3,4-dimethoxyphenyl)-morpholin-4-yl-methyl]-thiazolidine2,4-dione (4h)

IR [KBr r cm$\left.{ }^{-1}\right]: 3421.46(-\mathrm{NH}-), 1685.26(\mathrm{C}=\mathrm{O}), 1284.62(\mathrm{C}-\mathrm{N})$, 2979.85 (C-H), 3089.13 (=C-H), 620.45 (C-S), 1115.65 (C-O-C). ${ }^{1} \mathrm{H}-\mathrm{NMR}\left[400 \mathrm{MHz}, \delta, \mathrm{ppm}, \mathrm{DMSO}-d_{6}\right.$ ]: $12.264(\mathrm{~s}, 1 \mathrm{H}, \mathrm{NH}), 4.145-$ 4.189 (d, 1H, TZD CH), 4.374-4.485 (d, 1H, N-CH), 6.452 (s, 1H, 2'-H), 6.678-6.894 (d, 2H, 5'-H\&6'-H), 3.964 (s, 6H, 3'-OCH \& 4' $\mathrm{OCH}_{3}$ ), 3.745-3.865 (t, 4H, $\left.\mathrm{CH}_{2}-\mathrm{O}-\mathrm{CH}_{2}\right), 2.746-2.894\left(\mathrm{t}, 4 \mathrm{H}, \mathrm{CH}_{2}-\right.$ $\mathrm{N}-\mathrm{CH}_{2}$ ). ${ }^{13} \mathrm{C}-\mathrm{NMR}\left[400 \mathrm{MHz}, \delta, \mathrm{ppm}, \mathrm{DMSO}-d_{6}\right]: 177.88,169.46$, 153.56, 149.25, 132.45, 125.26, 118.24, 113.46, 69.05, 61.85, 58.47, 55.62, 50.43. ESI-MS: $\left(\mathrm{M}^{+}\right) \mathrm{m} / \mathrm{z} 352$.

5-[(3,4,5-trimethoxyphenyl)-morpholin-4-yl-methyl]thiazolidine-2,4-dione (4i)

IR $\left[\mathrm{KBr} \vee \mathrm{cm}^{-1}\right]: 3385.41(-\mathrm{NH}-), 1676.74(\mathrm{C}=\mathrm{O}), 1268.46(\mathrm{C}-\mathrm{N})$, $2959.74(\mathrm{C}-\mathrm{H}), 3094.25$ (=C-H), 628.71 (C-S), 1134.84 (C-O-C). ${ }^{1} \mathrm{H}-\mathrm{NMR}\left[400 \mathrm{MHz}, \delta, \mathrm{ppm}, \mathrm{DMSO}-\mathrm{d}_{6}\right.$ ]: 12.114 (s, $\left.1 \mathrm{H}, \mathrm{NH}\right), 4.058-$ 4.194 (d, 1H, TZD CH), 4.405-4.492 (d, 1H, N-CH), 6.248 (s, 2H, $2^{\prime}-\mathrm{H} \& 6^{\prime}-\mathrm{H}$ ), 3.954 (s, 9H, 3', 4', 5'-triOCH${ }_{3}$ ), 3.725-3.849 (t, 4H, $\left.\mathrm{CH}_{2}-\mathrm{O}-\mathrm{CH}_{2}\right), 2.652-2.845\left(\mathrm{t}, 4 \mathrm{H}, \mathrm{CH}_{2}-\mathrm{N}-\mathrm{CH}_{2}\right) \cdot{ }^{13} \mathrm{C}-\mathrm{NMR}[400$ $\mathrm{MHz}, \delta, \mathrm{ppm}, \mathrm{DMSO}-\mathrm{d}_{6}$ ]: 179.05, 168.19, 151.42, 140.74, 135.65, 112.27, 68.91, 63.64, 57.41, 53.44, 50.22. ESI-MS: $\left(M^{+}\right)$m/z 382.

5-[(4-hydroxy-3-methoxyphenyl)-morpholin-4-yl-methyl]thiazolidine-2,4-dione (4j)

IR [KBr r cm$\left.{ }^{-1}\right]: 3325.41(-\mathrm{NH}-), 1675.64(\mathrm{C}=\mathrm{O}), 1291.45(\mathrm{C}-\mathrm{N})$, $2980.65(\mathrm{C}-\mathrm{H}), 3091.47(=\mathrm{C}-\mathrm{H}), 622.11(\mathrm{C}-\mathrm{S}), 1125.62(\mathrm{C}-\mathrm{O}-\mathrm{C})$, $3512.22(\mathrm{Ph}-\mathrm{OH}) .{ }^{1} \mathrm{H}-\mathrm{NMR}\left[400 \mathrm{MHz}, \delta, \mathrm{ppm}, \mathrm{DMSO}-d_{6}\right.$ ]: 12.005 (s, $1 \mathrm{H}, \mathrm{NH}), 4.105-4.184(\mathrm{~d}, 1 \mathrm{H}, \mathrm{TZD} \mathrm{CH}), 4.357-4.462(\mathrm{~d}, 1 \mathrm{H}$, $\mathrm{N}-\mathrm{CH}), 6.348\left(\mathrm{~s}, 1 \mathrm{H}, 2^{\prime}-\mathrm{H}\right), 6.548-6.754\left(\mathrm{~d}, 1 \mathrm{H}, 5^{\prime}-\mathrm{H}\right), 6.874-$ $6.994\left(\mathrm{~d}, 1 \mathrm{H}, 6^{\prime}-\mathrm{H}\right), 9.548$ (s, 1H, $\mathrm{C}_{6} \mathrm{H}_{4}-\mathrm{OH}$ ), 3.895 (s, 3H, 3'$\mathrm{OCH}_{3}$ ), 3.765-3.910 (t, 4H, $\left.\mathrm{CH}_{2}-\mathrm{O}-\mathrm{CH}_{2}\right), 2.747-2.897\left(\mathrm{t}, 4 \mathrm{H}, \mathrm{CH}_{2}-\right.$ $\mathrm{N}-\mathrm{CH}_{2}$ ). ${ }^{13} \mathrm{C}-\mathrm{NMR}\left[400 \mathrm{MHz}, \delta, \mathrm{ppm}, \mathrm{DMSO}-d_{6}\right.$ ]: 175.44, 167.41, 155.22, 148.45, 131.58, 123.21, 119.55, 112.41, 68.08, 62.44, 59.75, 56.32, 50.85. ESI-MS: $\left(\mathrm{M}^{+}\right) \mathrm{m} / \mathrm{z} 338$.

5-[(4-bromophenyl)-morpholin-4-yl-methyl]-thiazolidine-2,4dione $(4 \mathrm{k})$

IR $\left[\mathrm{KBr} \mathrm{r} \mathrm{cm}^{-1}\right]: 3389.81(-\mathrm{NH}-), 1686.35(\mathrm{C}=\mathrm{O}), 1271.64(\mathrm{C}-\mathrm{N})$, $2992.67(\mathrm{C}-\mathrm{H}), 3089.45(=\mathrm{C}-\mathrm{H}), 621.12(\mathrm{C}-\mathrm{S}), 1131.83(\mathrm{C}-\mathrm{O}-\mathrm{C})$, 578.63 (C-Br). ${ }^{1} \mathrm{H}-\mathrm{NMR}$ [400 MHz, $\delta, \mathrm{ppm}, \mathrm{DMSO}-\mathrm{d}_{6}$ ]: 12.158 (s, $1 \mathrm{H}, \mathrm{NH}), 4.317-4.397$ (d, 1H, TZD CH), 4.135-4.187 (d, 1H, N-CH), 7.278-7.314 (d, 2H, 3'-H\&5'-H), 6.942-7.108 (d, 2H, 2'-H\&6'-H), 3.618-3.685 (t, 4H, CH$-\mathrm{O}-\mathrm{CH}_{2}$ ), 2.612-2.732 (t, 4H, $\mathrm{CH}_{2}-\mathrm{N}-\mathrm{CH}_{2}$ ). ${ }^{13} \mathrm{C}-\mathrm{NMR}\left[400 \mathrm{MHz}, \delta, \mathrm{ppm}, \mathrm{DMSO}-d_{6}\right.$ ]: 178.54, 166.64, 138.18, $133.79,130.52,122.15,66.35,57.72,53.23,50.48$. ESI-MS: $\left(M^{+}\right)$ $\mathrm{m} / \mathrm{z} 371$. 
5-[(3-bromophenyl)-morpholin-4-yl-methyl]-thiazolidine-2,4dione (4l)

IR [KBr r cm${ }^{-1}$ ]: $3412.54(-\mathrm{NH}-), 1678.75(\mathrm{C}=0), 1269.18(\mathrm{C}-\mathrm{N})$, $2987.2(\mathrm{C}-\mathrm{H}), 3086.23(=\mathrm{C}-\mathrm{H}), 619.84(\mathrm{C}-\mathrm{S}), 1128.46(\mathrm{C}-\mathrm{O}-\mathrm{C})$, 584.45 (C-Br). ${ }^{1} \mathrm{H}-\mathrm{NMR}$ [400 MHz, $\delta, \mathrm{ppm}, \mathrm{DMSO}-d_{6}$ ]: 11.846 (s, $1 \mathrm{H}, \mathrm{NH}), 4.365-4.381$ (d, $1 \mathrm{H}, \mathrm{TZD} \mathrm{CH}), 4.208-4.299$ (d, $1 \mathrm{H}$, $\mathrm{N}-\mathrm{CH}), 7.875\left(\mathrm{~s}, 1 \mathrm{H}, 2^{\prime}-\mathrm{H}\right), 7.457-7.510\left(\mathrm{~d}, 1 \mathrm{H}, 4^{\prime}-\mathrm{H}\right), 7.108-7.201$ $\left(\mathrm{t}, 1 \mathrm{H}, 5^{\prime}-\mathrm{H}\right), 6.847-6.912\left(\mathrm{~d}, 1 \mathrm{H}, 6^{\prime}-\mathrm{H}\right), 3.748-3.8414\left(\mathrm{t}, 4 \mathrm{H}, \mathrm{CH}_{2}-\right.$ O- $\left.\mathrm{CH}_{2}\right), 2.546-2.698\left(\mathrm{t}, 4 \mathrm{H}, \mathrm{CH}_{2}-\mathrm{N}-\mathrm{CH}_{2}\right) .{ }^{13} \mathrm{C}-\mathrm{NMR}[400 \mathrm{MHz}, \delta$, ppm, DMSO- $d_{6}$ ]: 175.51, 168.25, 139.17, 136.85, 133.79, 130.52, 126.62, 121.87, 68.55, 58.74, 54.76, 50.08. ESI-MS: $\left(M^{+}\right) \mathrm{m} / \mathrm{z} 371$.

5-[(4-dimethylaminophenyl)-morpholin-4-yl-methyl]thiazolidine-2,4-dione (4m)

IR [KBr r cm$~^{-1}$ : $3426.45(-\mathrm{NH}-), 1684.24(\mathrm{C}=\mathrm{O}), 1289.47(\mathrm{C}-\mathrm{N})$, 2992.35 (C-H), 3084.47 (=C-H), 627.74 (C-S), 1118.64 (C-O-C). 'H-NMR [400 MHz, $\delta, ~ p p m, ~ D M S O-d_{6}$ ]: 12.248 (s, 1H, NH), 4.462$4.510(\mathrm{~d}, 1 \mathrm{H}, \mathrm{TZD} \mathrm{CH}), 4.256-4.347(\mathrm{~d}, 1 \mathrm{H}, \mathrm{N}-\mathrm{CH}), 7.415-7.511$ (d, 1H, 2'-H\&6'-H), 6.917-7.125 (d, 1H, 3'-H\&5'-H), 3.148 (s, 6H, $\left.\mathrm{H}_{3} \mathrm{C}-\mathrm{N}-\mathrm{CH}_{3}\right), 3.839-3.990\left(\mathrm{t}, 4 \mathrm{H}, \mathrm{CH}_{2}-\mathrm{O}-\mathrm{CH}_{2}\right), 2.847-2.954$ (t, $4 \mathrm{H}, \mathrm{CH}_{2}-\mathrm{N}-\mathrm{CH}_{2}$ ). ${ }^{13} \mathrm{C}-\mathrm{NMR}\left[400 \mathrm{MHz}, \delta, \mathrm{ppm}, \mathrm{DMSO}-d_{6}\right.$ ]: 178.77 , 169.71, 152.82, 135.54, 129.64, 119.55, 68.74, 65.46, 59.44, 57.62, 43.25. ESI-MS: $\left(M^{+}\right) \mathrm{m} / \mathrm{z} 335$.

5-[(4-isopropylphenyl)-morpholin-4-yl-methyl]-thiazolidine2,4-dione (4n)

IR [KBr r cm-1]: $3386.62(-\mathrm{NH}-), 1693.24(\mathrm{C}=0), 1276.48(\mathrm{C}-\mathrm{N})$, $2989.42(\mathrm{C}-\mathrm{H}), 3090.48(=\mathrm{C}-\mathrm{H}), 613.75$ (C-S), 1125.64 (C-O-C). 'H-NMR [400 MHz, $\delta, p p m$, DMSO- $d_{6}$ ]: 12.156 (s, 1H, NH), 4.3244.398 (d, 1H, TZD CH), 4.158-4.205 (d, 1H, N-CH), 7.146-7.205 (d, 1H, 2'-H\&6'-H), 7.452-7.512 (d, 1H, 3'-H\&5'-H), 2.421-2.564 (dd, $6 \mathrm{H}, \underline{\mathrm{H}}_{3} \mathrm{C}-\mathrm{CH}-\mathrm{CH}_{3}$ ), 2.875-3.105 (m, 1H, $\mathrm{H}_{3} \mathrm{C}-\mathrm{CH}-\mathrm{CH}_{3}$ ), 3.7543.897 (t, 4H, CH $\left.\mathrm{CH}_{2}-\mathrm{O}-\mathrm{CH}_{2}\right), 2.759-2.851\left(\mathrm{t}, 4 \mathrm{H}, \mathrm{CH}_{2}-\mathrm{N}-\mathrm{CH}_{2}\right.$ ). ${ }^{13} \mathrm{C}-\mathrm{NMR}$ [400 MHz, $\delta, \mathrm{ppm}$, DMSO- $d_{6}$ ]: 174.32, 168.46, 150.41, 136.52, 129.22, 122.48, 67.71, 64.41, 58.32, 54.40, 35.46, 27.56. ESI-MS: $\left(M^{+}\right) \mathrm{m} / \mathrm{z} 334$.

\section{Biological evaluation}

\section{In vivo hypoglycemic activity screening}

Wistar albino rats of both sexes weighing 160-200 g for acute and chronic studies were purchased from Sainadh Agencies Laboratory animal suppliers, Hyderabad. Acute and chronic studies were carried out on alloxan induced Wistar albino rats by tail tipping method. ${ }^{24,25}$ The rats were acclimatized for a week to the normal laboratory conditions prior to commencing the experiments, with ad libitum access to tap water and pellets. The rats were housed in cages with $12 \mathrm{~h} / 12 \mathrm{~h}$ dark and light cycle at room temperature. Intraperitoneally alloxan monohydrate $120 \mathrm{mg} / \mathrm{kg}$ in normal saline was administered to the acclimatized animals, kept fasting for $24 \mathrm{~h}$ with water ad libitum. To overcome the early hypoglycemic phase, $5 \%$ dextrose solution was given for a day. After $72 \mathrm{~h}$, by tail tipping method a drop of blood from the tail vein was collected and blood glucose levels as well as biochemical parameters were measured using a digital Accu-Chek active digital glucometer and Robonik biochemical analyzer, respectively. Rats having blood glucose levels above $150 \mathrm{mg} / \mathrm{dL}$ were selected for the study and divided into six groups. For the acute study, $36 \mathrm{mg} / \mathrm{kg}$ body weight dose was calculated by considering thiazolidinedione derivatives equivalent to average human intake $200 \mathrm{mg} / \mathrm{kg}$. The test compounds were given orally by mixing with CMC$0.25 \%$ solution. Rosiglitazone at $30 \mathrm{mg} / \mathrm{kg}$ body weight dose was used as the standard drug. At $0 \mathrm{~h}, 1 \mathrm{~h}, 2 \mathrm{~h}, 4 \mathrm{~h}, 6 \mathrm{~h}$, and $8 \mathrm{~h}$ blood samples were withdrawn and analyzed for blood glucose level. Based on the acute study results limited compounds were selected for the chronic study. Doses of 35 and $70 \mathrm{mg} / \mathrm{kg}$ body weight were taken into consideration in the chronic study. On day 7 and day 15, decrease in blood glucose was observed by measuring the blood glucose levels.

\section{In vitro anti-inflammatory activity screening}

In vitro anti-inflammatory activity was evaluated by human red blood cell (HRBC) membrane stabilization method and protein denaturation method. ${ }^{26-28}$

\section{HRBC membrane stabilization method}

Fresh whole blood was collected from a healthy human volunteer who had not used any nonsteroidal anti-inflammatory drug 2 weeks prior to the experiment and mixed with an equal volume of sterilized Alsever's solution ( $0.8 \%$ sodium citrate, $2 \%$ dextrose, $0.42 \%$ sodium chloride, and $0.05 \%$ citric acid in water). At $3000 \mathrm{rpm}$ the blood was centrifuged for $10 \mathrm{~min}$ and the packed cells were washed three times with $0.85 \%$ isosaline (pH 7.2). The volume of blood was measured and reconstituted with isosaline as $10 \% \mathrm{v} / \mathrm{v}$ suspension. Different concentrations $(50,100,300$, and $500 \mu \mathrm{g} / \mathrm{mL}$ ) of the test solutions were prepared in isosaline. To $1 \mathrm{~mL}$ of test solution were added 2 $\mathrm{mL}$ of hyposaline $(0.25 \% \mathrm{w} / \mathrm{v} \mathrm{NaCl}), 1 \mathrm{~mL}$ of $0.15 \mathrm{M}$ phosphate buffer ( $\mathrm{pH} 7.4$ ), and $0.5 \mathrm{~mL}$ of $10 \%$ HRBC in isosaline. For the test control, $1 \mathrm{~mL}$ of distilled water used instead of hyposaline, while the product control lacked red blood cells. The mixtures were incubated at $37^{\circ} \mathrm{C}$ for $30 \mathrm{~min}$ and centrifuged at $3000 \mathrm{rpm}$ for about $20 \mathrm{~min}$. Diclofenac sodium $(100 \mu \mathrm{g} / \mathrm{mL}$ and $200 \mu \mathrm{g} /$ $\mathrm{mL}$ ) was used as the reference drug. The hemoglobin content in the suspension was estimated using a UV-spectrophotometer at $560 \mathrm{~nm}$. Percentage inhibition activity was calculated as follows:

$\%$ inhibition $=100-\left[\frac{\mathrm{OD} \text { of test }}{\mathrm{OD} \text { of control }} \times 100\right]$

where optical density (OD) of test sample is OD or absorbance of the test sample absorbance and $\mathrm{OD}$ of control is $\mathrm{OD}$ or absorbance of the negative control.

\section{Protein denaturation method}

Denaturation of proteins is a well-documented cause of inflammation. Salicylic acid, phenylbutazone, diclofenac, and flufenamic acid are anti-inflammatory drugs that have shown dose dependent ability for thermally induced protein denaturation. Agents that can prevent protein denaturation would be advisable for anti-inflammatory drug development. Each $0.5 \mathrm{~mL}$ of test sample solution consists of $0.45 \mathrm{~mL}$ of $5 \%$ $\mathrm{w} / \mathrm{v}$ aqueous bovine serum albumin solution and $0.05 \mathrm{~mL}$ of test sample of different concentrations $(50,100,300$, and 500 
$\mu \mathrm{g} / \mathrm{mL}$ ), while $0.5 \mathrm{~mL}$ of test control solution consists of 0.45 $\mathrm{mL}$ of $5 \% \mathrm{w} / \mathrm{v}$ aqueous bovine serum albumin solution and 0.05 $\mathrm{mL}$ of distilled water, and $0.5 \mathrm{~mL}$ of standard solution consists of $0.45 \mathrm{~mL}$ of $5 \% \mathrm{w} / \mathrm{v}$ aqueous bovine serum albumin solution and $0.05 \mathrm{~mL}$ of different concentrations (100 and $200 \mu \mathrm{g} / \mathrm{mL}$ ) of diclofenac sodium. All the solutions were adjusted to $\mathrm{pH} 6.3$ using $1 \mathrm{~N} \mathrm{HCl}$. The samples were incubated at $37^{\circ} \mathrm{C}$ for $20 \mathrm{~min}$ and the temperature was raised to keep the samples at $57^{\circ} \mathrm{C}$ for $3 \mathrm{~min}$. After cooling, $2.5 \mathrm{~mL}$ of phosphate buffer was added to the above solutions. The absorbance was measured using a UV-visible spectrophotometer at $600 \mathrm{~nm}$. The percentage inhibition of protein denaturation was calculated as follows:

$$
\% \text { inhibition }=100-\left[\left\{\frac{\mathrm{OD} \text { of test }-\mathrm{OD} \text { of product control }}{\mathrm{OD} \text { of test control }}\right\} \times 100\right]
$$

\section{MOLECULAR DOCKING}

Docking studies were performed to identify novel insulin sensitizers by observing the molecular interactions of designed ligands with PPAR $\gamma$ receptor protein and to determine the potential anti-inflammatory agents at cyclooxygenase (COX)-1 and COX2 isoenzymes. The selection of the target protein for docking is based on several factors such as it must possess resolution between 2.0 and $3.0 \AA$, the structure should be determined by $X$-ray diffraction, it consists of co-crystallized ligand, and it does not have any protein breaks in the selected protein's 3D structure. PPARy receptor protein is the most promising target for the identification of antidiabetic drugs possessing a thiazolidinedione nucleus. ${ }^{29,30}$ The crystal structure of the PPAR $y$ target receptor protein was obtained from the protein databank PDB ID: 2PRG having resolution of $2.3 \AA$. COX isoenzymes were the targets for determining the anti-inflammatory activity and the target proteins were downloaded from a protein databank PDB ID: 1EQG (COX-1) and PDB ID: 1CX2 (COX-2) having a resolution of $2.6 \AA$ and $3.0 \AA$, respectively. ${ }^{31}$ AutoDock 4.2 .6 software was utilized to know the type of interactions of the designed 3D-structured thiazolidinediones with the 2PRG, 1EQG, and 1CX2 active site regions. ChemDraw Ultra 8.0 software was used to draw the designed structures and they were converted into suitable 3D models. By applying molecular mechanics they were subjected to energy minimizations, which are required for molecular docking and for the preparation of corresponding pdb files. Docking studies were preformed to find out the possible locations for the ligand in the active site region of the receptor. Grid-based docking studies was carried out using default parameters and docking was performed by considering rosiglitazone, indomethacin, and celecoxib as standard ligands at PPAR $\gamma$ receptor protein, COX-1, and COX-2 active sites, respectively.

\section{RESULTS AND DISCUSSION}

\section{Chemistry}

Initially TZD (3) was synthesized conventionally, which on further reaction with various substituted aromatic aldehydes and morpholine as secondary amines underwent the Mannich reaction to yield the series of designed and title Mannich bases represented in Scheme 1. TZD (3) and the title Mannich base compounds $4 \mathrm{a}-4 \mathrm{n}$ were also prepared by microwave-assisted irradiation with $280 \mathrm{~W}$ and $420 \mathrm{~W}$ power levels at different steps of the synthesis. The physical characterization data, the comparative studies of conventional synthesis and microwave irradiation synthesis with respect to percentage yields, and their reaction time intervals are shown in Table 1.

Table 1. Physical characterization data of synthesized compounds $4 \mathrm{a}-4 \mathrm{n}$

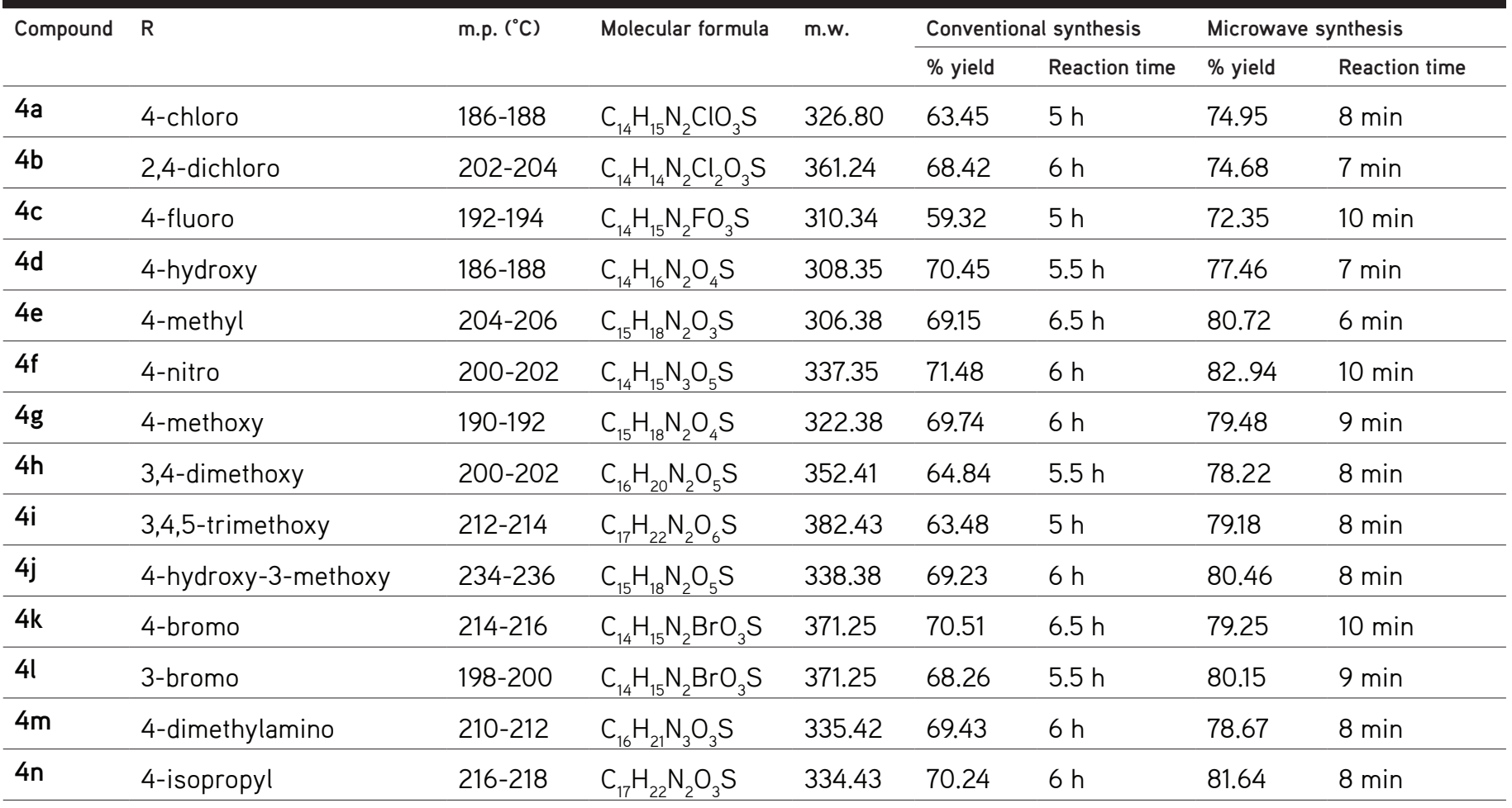


The designed and synthesized Mannich bases $4 a-4 n$ were confirmed by the IR absorption bands at $3300-3500 \mathrm{~cm}^{-1}$ characteristic of $-\mathrm{NH}$ - of TZD, $1640-1690 \mathrm{~cm}^{-1}$ characteristic of $-\mathrm{C}=\mathrm{O}$ of TZD, $3000-3100 \mathrm{~cm}^{-1}$ characteristic of $=\mathrm{C}-\mathrm{H}$ stretching of the aromatic phenyl ring, and $1050-1300 \mathrm{~cm}^{-1}$ characteristic of $\mathrm{C}-\mathrm{O}$ stretching of morpholine. The ${ }^{1} \mathrm{H}-\mathrm{NMR}$ spectra produced a singlet at 11-12.5 ppm that is characteristic of the $\mathrm{NH}$ proton of TZD, triplets at 2-3.8 ppm indicating methylene group protons of morpholine, doublets at 4-4.5 ppm indicating $\mathrm{CH}$ of TZD, and $\mathrm{N}-\mathrm{CH}$ protons, aromatic protons, were exhibited at 6.5-8 ppm. In ${ }^{13} \mathrm{C}-\mathrm{NMR}$, the signal appeared at $165-200 \mathrm{ppm}$ indicating $\mathrm{C}=\mathrm{O}$ of TZD, signals at $120-160 \mathrm{ppm}$ indicate aromatic carbons, 10-50 ppm signals indicate aliphatic carbons, and 30-60 ppm signals indicate $-\mathrm{CH}_{2}-\mathrm{N}-\mathrm{CH}_{2}$ - carbons.

\section{In vivo hypoglycemic activity}

In vivo hypoglycemic activity study protocols were approved by the Institutional Animal Ethics Committee under the supervision of Committee for the Purpose of Control and Supervision of Experiments on Animals (CPCSEA), New Delhi, bearing registration number: 1847/PO/Re/S/16/CPCSEA. Blood glucose levels and body weights were expressed as mean \pm standard error of the mean (SEM). The values were analyzed by one-way analysis of variance followed by Dunnet's " $t$ " test and they were considered significant when compared to the normal group. The acute and chronic study results are given in Tables 2 and 3 , respectively. The acute study results showed compounds $4 \mathrm{c}$, 4h, and $4 \mathrm{n}$ exhibited good hypoglycemic activity and they were subjected to the chronic study at 35 and $70 \mathrm{mg} / \mathrm{kg}$ body weight. The chronic study results indicate that compounds $4 \mathrm{~h}$ and $4 \mathrm{n}$ exhibited good activity at a dose of $70 \mathrm{mg} / \mathrm{kg}$ body weight.

In vitro anti-inflammatory activity

As a part of the in vitro anti-inflammatory activity investigation, the compounds were tested for HRBC membrane stabilization and protein denaturation by taking diclofenac sodium as<smiles>O=C(O)CCl</smiles>

(1)<smiles>NC(=S)S</smiles>

(2)

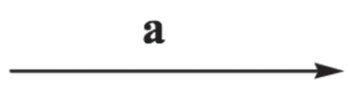<smiles>O=C1CSC(=O)N1</smiles>

(3)
$\mathbf{R}=$ 4a: 4-chrolo
4b: 2,4-dichloro
4c: 4-fluoro
4d: 4-hydroxy
4e: 4-methyl
4f: 4-nitro
4g: 4-methoxy

4h: 3,4-dimethoxy

4i: 3,4,5-trimethoxy

4j: 4-hydroxy-3-methoxy

4k: 4-bromo

4l: 3-bromo

4m: 4-dimethylamino

4n: 4-isopropyl

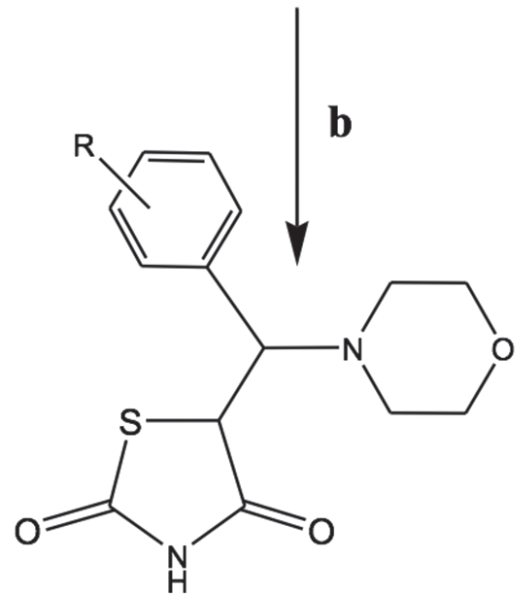

4 (a-k)

Conventional synthesis: a) $\mathrm{H}_{2} \mathrm{O}$, Conc. $\mathrm{HCl}, 100-110^{\circ} \mathrm{C}, 10-12 \mathrm{hrs}$

b) aromatic aldehyde, morpholine, ethanol, Conc. HCl, 5-6 hrs

Microwave irradiation: a) $\mathrm{H}_{2} \mathrm{O}$, Conc. $\mathrm{HCl}, 280 \mathrm{~W}, 120^{\circ} \mathrm{C}, 6 \mathrm{~min}$

b) aromatic aldehyde, morpholine, ethanol, Conc. $\mathrm{HCl}, 420 \mathrm{~W}, 8-10 \mathrm{~min}$ 
standard. Percentage inhibition of test compounds at various concentration levels was calculated and the results are given in Tables 4 and 5. HRBC membrane stabilization indicated the standard drug diclofenac sodium showed $90.78 \pm 0.87 \%$ inhibition at $300 \mu \mathrm{g} / \mathrm{mL}$ concentration. The highest inhibition of $90.64 \pm 0.26 \%$ and $89.61 \pm 0.25 \%$ was exhibited by compounds $4 \mathrm{k}$ and $4 \mathrm{f}$, respectively, at $500 \mu \mathrm{g} / \mathrm{mL}$ concentration. Protein denaturation indicated the standard drug diclofenac sodium showed $95.34 \pm 0.92 \%$ inhibition at $300 \mu \mathrm{g} / \mathrm{mL}$ concentration. In protein denaturation, the highest inhibition of $93.72 \pm 0.61 \%$ was exhibited by compound $4 \mathrm{k}$ at $500 \mu \mathrm{g} / \mathrm{mL}$ concentration. All the values were expressed as mean \pm SEM, $n=3$.

\section{Molecular docking}

Molecular docking studies at PPAR $\gamma$ receptor protein, COX-1, and COX-2 active site regions give the data of binding energy ( $\mathrm{kcal} / \mathrm{moL}$ ), number of hydrogen bonds, hydrogen bond length, and interacted amino acid residues, shown in Tables 6, 7, and 8. In comparison with the standard ligand rosiglitazone (binding energy $-8.26 \mathrm{kcal} / \mathrm{moL}$ ), compounds $4 \mathrm{~h}$ and $4 \mathrm{n}$ showed higher

Table 2. Effect of synthesized compounds $3 a-31$ on blood glucose level in alloxan induced diabetic rats (acute study)

\begin{tabular}{|c|c|c|c|c|c|c|}
\hline Compound & $\mathrm{Oh}$ & $1 \mathrm{~h}$ & $2 \mathrm{~h}$ & $4 \mathrm{~h}$ & $6 \mathrm{~h}$ & $8 \mathrm{~h}$ \\
\hline Normal & $121.24 \pm 2.56$ & $123.64 \pm 2.04$ & $122.5 \pm 5.11$ & $121.54 \pm 3.47$ & $120.5 \pm 4.22$ & $120.33 \pm 2.3$ \\
\hline Standard & $398.26 \pm 4.22^{*}$ & $242.34 \pm 5.48$ & $192.1 \pm 4.29^{*}$ & $148.64 \pm 3.46^{*}$ & $110.35 \pm 4.65$ & $100.55 \pm 5.26^{* *}$ \\
\hline $4 a$ & $326.45 \pm 2.64$ & $294.32 \pm 4.41^{* *}$ & $265.61 \pm 4.68$ & $250.31 \pm 8.61$ & $253.12 \pm 8.32^{* *}$ & $290.5 \pm 4.85$ \\
\hline $4 b$ & $315.22 \pm 8.15^{\star *}$ & $254.26 \pm 6.78$ & $235.81 \pm 6.34^{*}$ & $175.52 \pm 3.51$ & $165.45 \pm 5.61^{*}$ & $200.61 \pm 2.46$ \\
\hline $4 c$ & $325.64 \pm 8.55$ & $249.6 \pm 5.44^{* *}$ & $199.72 \pm 7.14^{*}$ & $150.4 \pm 6.45$ & $118.12 \pm 6.47^{\star *}$ & $125.47 \pm 3.84$ \\
\hline $4 d$ & $322.3 \pm 4.22$ & $299.52 \pm 6.38 *$ & $235.42 \pm 4.28^{* *}$ & $168.5 \pm 2.64$ & $154.8 .3 \pm 6.55$ & $198.24 \pm 5.24^{\star *}$ \\
\hline $4 e$ & $315.88 \pm 5.64$ & $305.11 \pm 7.34$ & $296.58 \pm 5.42$ & $235.25 \pm 2.65^{\star *}$ & $258.44 \pm 6.54$ & $272.05 \pm 7.56$ \\
\hline $4 f$ & $305.44 \pm 5.26^{* *}$ & $297.53 \pm 6.48$ & $285 \pm 7.12^{*}$ & $252.56 \pm 5.29$ & $278.32 \pm 5.48^{*}$ & $308 \pm 6.24^{*}$ \\
\hline $4 g$ & $316.0 \pm 8.54^{*}$ & $291.5 \pm 6.44^{*}$ & $252.5 \pm 4.62$ & $230.45 \pm 4.69$ & $268.2 \pm 5.48^{*}$ & $274.68 \pm 8.47$ \\
\hline $4 \mathrm{~h}$ & $335.78 \pm 8.95$ & $250.55 \pm 4.37^{\star *}$ & $200.48 \pm 5.48^{* *}$ & $153.51 \pm 4.62^{* *}$ & $112.4 \pm 5.12$ & $125.42 \pm 5.46$ \\
\hline $4 \mathrm{i}$ & $334.54 \pm 4.18^{*}$ & $309.85 \pm 4.68^{* *}$ & $295.23 \pm 6.18$ & $254 \pm 6.47^{*}$ & $289.14 \pm 5.61^{*}$ & $306.47 \pm 5.28$ \\
\hline $4 j$ & $309.52 \pm 2.84$ & $300.15 \pm 6.15^{\star}$ & $285.3 \pm 7.52$ & $265.9 \pm 4.28^{\star *}$ & $273.58 \pm 4.35^{\star \star}$ & $298.09 \pm 5.51$ \\
\hline $4 k$ & $338.4 \pm 6.41^{*}$ & $310.68 \pm 3.48$ & $279.5 \pm 6.45^{\star *}$ & $254.30 \pm 5.49$ & $281.21 \pm 5.41$ & $298.45 \pm 6.32^{\star *}$ \\
\hline 41 & $325.64 \pm 2.54$ & $308 \pm 5.48$ & $285.46 \pm 8.17$ & $265.48 \pm 6.48$ & $285.15 \pm 5.48^{* *}$ & $310.56 \pm 4.29^{*}$ \\
\hline $4 m$ & $319.25 \pm 5.35^{*}$ & $318.62 \pm 4.65$ & $294.32 \pm 6.21^{*}$ & $268.48 \pm 7.15^{\text {** }}$ & $294.54 \pm 4.62$ & $300.58 \pm 5.42^{\star *}$ \\
\hline $4 n$ & $320.61 \pm 5.16^{* *}$ & $260.46 \pm 3.16$ & $210.45 \pm 4.65^{\star}$ & $145.68 \pm 5.49$ & $120.64 \pm 4.68$ & $130.64 \pm 6.48^{*}$ \\
\hline
\end{tabular}

All values expressed as mean \pm standard error of the mean, $n=6$. Standard drug: Rosiglitazone; Statistical analysis is done by one-way One-Way Analysis of Variance followed by Dunnet's " $t$ " test; ** $p<0.01$ (considered significant when compared to normal group), " $p<0.001$.

SEM: Standard error of the mean

Table 3. Effect of synthesized compounds $4 \mathrm{c}$, $4 \mathrm{~h}$, and $4 \mathrm{n}$ on fasting blood glucose level and body weight in alloxan induced diabetic rats (chronic study 15 days)

\begin{tabular}{|c|c|c|c|c|c|c|}
\hline \multirow[t]{2}{*}{ Compound } & \multicolumn{3}{|c|}{ Blood glucose in $\mathrm{mg} / \mathrm{dL}$} & \multicolumn{3}{|c|}{ Body weight in $\mathrm{g}$} \\
\hline & Day 0 & Day 7 & Day 15 & Day 0 & Day 7 & Day 15 \\
\hline Standard & $368.25 \pm 4.24$ & $200.65 \pm 3.63$ & $178.91 \pm 5.61$ & $196.5 \pm 4.27$ & $193.22 \pm 3.25$ & $194.2 \pm 5.24$ \\
\hline $4 \mathrm{c}(70 \mathrm{mg} / \mathrm{kg} \mathrm{bw})$ & $327.18 \pm 4.41$ & $228.46 \pm 3.54^{*}$ & $209.16 \pm 3.28$ & $199.31 \pm 2.64^{*}$ & $197.22 \pm 2.26^{*}$ & $195.21 \pm 2.77$ \\
\hline 4h (35 mg/kg bw) & $336.64 \pm 4.22^{* *}$ & $250.61 \pm 2.64$ & $228.42 \pm 5.16$ & $196.31 \pm 4.14$ & $198.47 \pm 2.67^{\star \star}$ & $195.44 \pm 3.12$ \\
\hline 4n (35 mg/kg bw) & $320.38 \pm 4.55$ & $254.35 \pm 3.14$ & $211.28 \pm 2.45$ & $198.66 \pm 2.61^{\star}$ & $199.34 \pm 2.61$ & $195.24 \pm 2.34^{*}$ \\
\hline 4n (70 mg/kg bw) & $320.64 \pm 3.27$ & $206.37 \pm 2.48^{* *}$ & $180.55 \pm 3.22^{* *}$ & $200.61 \pm 3.64$ & $198.46 \pm 3.48$ & $197.41 \pm 4.54$ \\
\hline
\end{tabular}

All values expressed as mean \pm standard error of the mean, $n=6$. Standard drug: rosiglitazone; Statistical analysis is done by one-way One-Way Analysis of Variance followed by Dunnet's " $t$ " test; ** $p<0.01$ (considered significant when compared to normal group), * $p<0.001$. 
Table 4. Effect of compounds $4 a-4 n$ on human red blood cell membrane stabilization

\begin{tabular}{lllll} 
Compound & \multicolumn{4}{l}{$\%$ Inhibition at different concentrations } \\
\cline { 2 - 5 } & $50 \mu \mathrm{g} / \mathrm{mL}$ & $100 \mu \mathrm{g} / \mathrm{mL}$ & $300 \mu \mathrm{g} / \mathrm{mL}$ & $500 \mu \mathrm{g} / \mathrm{mL}$ \\
\hline $\begin{array}{l}\text { Diclofenac } \\
\text { sodium }\end{array}$ & ---- & $86.79 \pm 0.43$ & $90.78 \pm 0.87$ & ----- \\
\hline $4 \mathrm{a}$ & $53.33 \pm 0.38$ & $58.67 \pm 0.32$ & $61.33 \pm 0.46$ & $68.00 \pm 0.62$ \\
\hline $4 \mathrm{~b}$ & $37.33 \pm 0.18$ & $48.00 \pm 0.46$ & $52.67 \pm 0.22$ & $65.33 \pm 0.32$ \\
\hline $4 \mathrm{c}$ & $42.67 \pm 0.32$ & $52.00 \pm 0.62$ & $61.33 \pm 0.28$ & $68.00 \pm 0.32$ \\
\hline $4 \mathrm{~d}$ & $46.00 \pm 0.32$ & $54.67 \pm 0.22$ & $65.33 \pm 0.46$ & $72.00 \pm 0.52$ \\
\hline $4 \mathrm{e}$ & $40.00 \pm 0.32$ & $46.00 \pm 0.52$ & $52.00 \pm 0.36$ & $54.00 \pm 0.42$ \\
\hline $4 \mathrm{f}$ & $64.25 \pm 0.45$ & $82.62 \pm 0.40$ & $85.46 \pm 0.32$ & $89.61 \pm 0.25$ \\
\hline $4 \mathrm{~g}$ & $52.34 \pm 0.36$ & $61.48 \pm 0.85$ & $72.42 \pm 0.60$ & $85.82 \pm 0.46$ \\
\hline $4 \mathrm{~h}$ & $58.64 \pm 0.42$ & $70.84 \pm 0.23$ & $76.55 \pm 0.44$ & $81.12 \pm 0.52$ \\
\hline $4 \mathrm{i}$ & $51.65 \pm 0.75$ & $60.33 \pm 0.48$ & $71.64 \pm 0.68$ & $78.45 \pm 0.72$ \\
\hline $4 \mathrm{j}$ & $49.25 \pm 0.24$ & $58.64 \pm 0.44$ & $65.98 \pm 0.52$ & $74.23 \pm 0.75$ \\
\hline $4 \mathrm{k}$ & $61.42 \pm 0.61$ & $80.43 \pm 0.64$ & $84.28 \pm 0.48$ & $90.64 \pm 0.26$ \\
\hline $4 \mathrm{l}$ & $54.40 \pm 0.44$ & $63.44 \pm 0.74$ & $71.68 \pm 0.80$ & $78.71 \pm 0.64$ \\
\hline $4 \mathrm{~m}$ & $58.64 \pm 0.62$ & $65.49 \pm 0.82$ & $70.18 \pm 0.42$ & $76.48 \pm 0.34$ \\
\hline $4 \mathrm{n}$ & $57.82 \pm 0.48$ & $62.47 \pm 0.62$ & $72.14 \pm 0.54$ & $78.91 \pm 0.46$ \\
\hline & & & & \\
\hline
\end{tabular}

All values expressed as mean \pm standard error of the mean, $n=3$

Table 5. Effect of compounds $4 \mathrm{a}-4 \mathrm{n}$ on protein denaturation

\begin{tabular}{lllll} 
Compound & \multicolumn{4}{l}{$\%$ Inhibition at different concentrations } \\
\cline { 2 - 5 } & $50 \mu \mathrm{g} / \mathrm{mL}$ & $100 \mu \mathrm{g} / \mathrm{mL}$ & $300 \mu \mathrm{g} / \mathrm{mL}$ & $500 \mu \mathrm{g} / \mathrm{mL}$ \\
\hline $\begin{array}{l}\text { Diclofenac } \\
\text { sodium }\end{array}$ & ---- & $88.89 \pm 0.36$ & $95.34 \pm 0.92$ & ---- \\
\hline $4 \mathrm{a}$ & $80.27 \pm 0.20$ & $83.47 \pm 0.35$ & $86.80 \pm 0.15$ & $88.80 \pm 0.42$ \\
\hline $4 \mathrm{~b}$ & $66.53 \pm 0.28$ & $84.53 \pm 0.42$ & $86.00 \pm 0.35$ & $86.80 \pm 0.45$ \\
\hline $4 \mathrm{c}$ & $54.67 \pm 0.18$ & $61.33 \pm 0.12$ & $71.93 \pm 0.45$ & $84.93 \pm 0.13$ \\
\hline $4 \mathrm{~d}$ & $61.33 \pm 0.46$ & $71.80 \pm 0.35$ & $78.67 \pm 0.38$ & $86.00 \pm 0.28$ \\
\hline $4 \mathrm{e}$ & $71.47 \pm 0.35$ & $78.60 \pm 0.42$ & $82.93 \pm 0.36$ & $86.80 \pm 0.25$ \\
\hline $4 \mathrm{f}$ & $83.42 \pm 0.35$ & $86.49 \pm 0.28$ & $87.42 \pm 0.33$ & $94.82 \pm 0.44$ \\
\hline $4 \mathrm{~g}$ & $78.64 \pm 0.54$ & $82.66 \pm 0.42$ & $88.48 \pm 0.40$ & $91.62 \pm 0.64$ \\
\hline $4 \mathrm{~h}$ & $81.80 \pm 0.24$ & $86.48 \pm 0.22$ & $88.64 \pm 0.86$ & $92.75 \pm 0.32$ \\
\hline $4 \mathrm{i}$ & $74.63 \pm 0.26$ & $80.46 \pm 0.26$ & $87.42 \pm 0.34$ & $90.66 \pm 0.28$ \\
\hline $4 \mathrm{j}$ & $78.62 \pm 0.42$ & $81.66 \pm 0.48$ & $86.94 \pm 0.55$ & $90.48 \pm 0.36$ \\
\hline $4 \mathrm{k}$ & $81.26 \pm 0.32$ & $85.46 \pm 0.46$ & $90.46 \pm 0.22$ & $93.72 \pm 0.61$ \\
\hline $4 \mathrm{l}$ & $81.46 \pm 0.62$ & $85.92 \pm 0.68$ & $88.33 \pm 0.82$ & $92.64 \pm 0.46$ \\
\hline $4 \mathrm{~m}$ & $72.61 \pm 0.64$ & $79.45 \pm 0.40$ & $84.24 \pm 0.28$ & $88.49 \pm 0.62$ \\
\hline $4 \mathrm{n}$ & $69.46 \pm 0.52$ & $72.24 \pm 0.64$ & $76.18 \pm 0.38$ & $82.81 \pm 0.12$ \\
\hline $\mathrm{Aln}$ & & & & \\
\hline
\end{tabular}

All values expressed as mean \pm standard error of the mean, $n=3$ binding energy $(-8.32 \mathrm{kcal} / \mathrm{moL}$ and $-8.29 \mathrm{kcal} / \mathrm{moL})$ at the active site region of PPAR $\gamma$ receptor protein. Figure 1 depicts the $3 D$ structure of PPAR $\gamma$ receptor protein, binding mode of rosiglitazone and compounds $4 \mathrm{~h}$ and $4 \mathrm{n}$ at the active site region of PPAR $\gamma$ protein (PDB ID: 2PRG). Compound 4k gave the highest binding energy $(-7.47 \mathrm{kcal} / \mathrm{moL}$ and $-10.98 \mathrm{kcal} / \mathrm{moL})$ with the two COX targets used in comparison with the binding energy of the standard ligands indomethacin $(-7.14 \mathrm{kcal} / \mathrm{moL})$ and celecoxib ( $-10.89 \mathrm{kcal} / \mathrm{moL})$. Figure 2 depicts the binding mode of indomethacin at the active site region of COX-1 (PDB ID: $1 E Q G$ ), celecoxib at the active site region of COX-2 (PDB ID: $1 \mathrm{CX} 2$ ), and compound $4 \mathrm{k}$ interaction with the two targets.

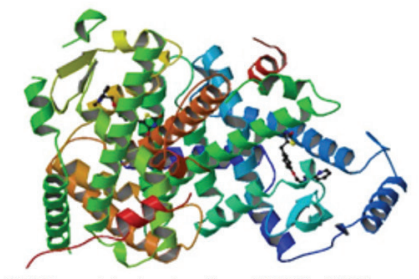

PPAR $y$ protein structure from PDB ID: 2PRG

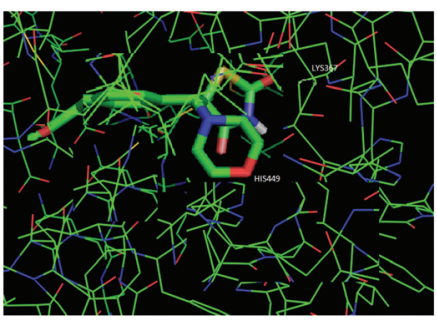

Binding mode of compound $4 \mathrm{~h}$ at active site region of PPAR $y$ protein PDB ID: 2PRG

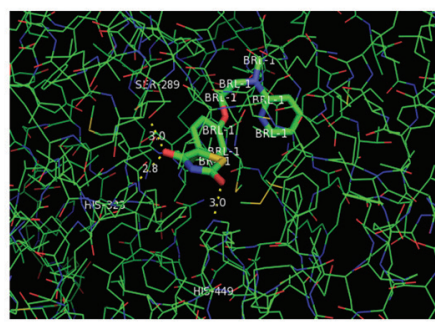

Binding mode of rosiglitazone at active site region of PPAR $\gamma$ protein PDB ID: 2PRG

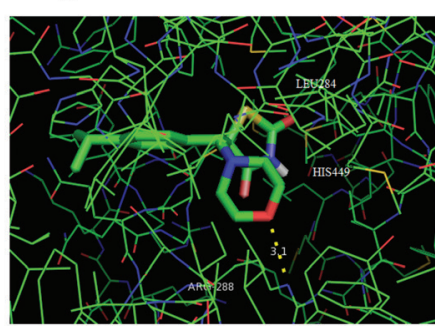

Binding mode of compound $4 \mathrm{n}$ at active site region of PPAR $\gamma$ protein PDB ID: 2 PRG
Figure 1. Molecular docking studies at active site region of PPAR $\gamma$ protein receptor (PBD ID: 2PRG)

PPAR $\gamma$ : Proliferator-activated receptor gamma

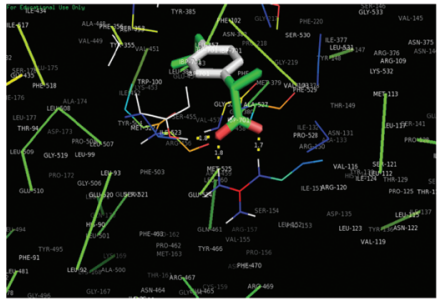

Binding mode of indomethacin at active site region of COX-1 PDB ID: 1EQG

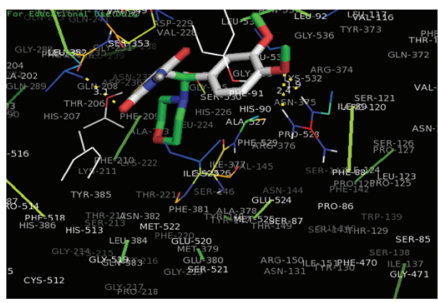

Binding mode of compound $4 \mathrm{k}$ at active site region of COX-1 PDB ID: 1EQG

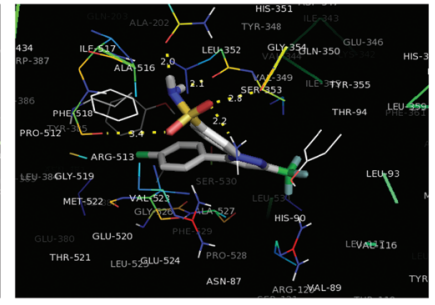

Binding mode of celecoxib at active site region of COX-2 PDB ID: 1CX2

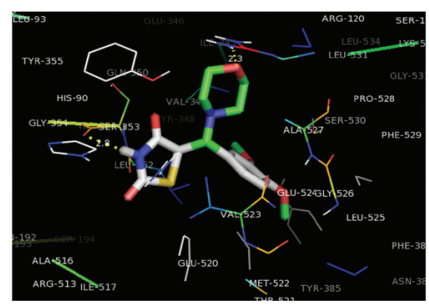

Binding mode of compound $4 \mathrm{k}$ at active site region of COX-2 PDB ID: 1CX2
Figure 2. Molecular docking studies at active site region of COX isoenzymes (COX-1 PBD ID: 1EQG \& COX-2 PDB ID: 1CX2) 


\section{CONCLUSION}

In this investigation various thiazolidinedione derivatives possessing morpholine and substituted phenyl appendages that are attached to the common methyl group were developed as Mannich bases by simple Mannich reaction. The title compounds were synthesized by conventional as well as microwave-assisted synthesis. Microwave irradiation produced high yield in a shorter reaction time in comparison with traditional conventional heating synthesis. Characterization of the compounds was done physically and spectrally. They were evaluated for in vivo hypoglycemic activity and in vitro antiinflammatory activity because of the existing thiazolidinedione

\section{Table 6. Binding energy and amino acid residues interacted by the compounds $4 a-4 n$ with the target PPAR $\gamma$ protein (PDB ID - 2PRG)}

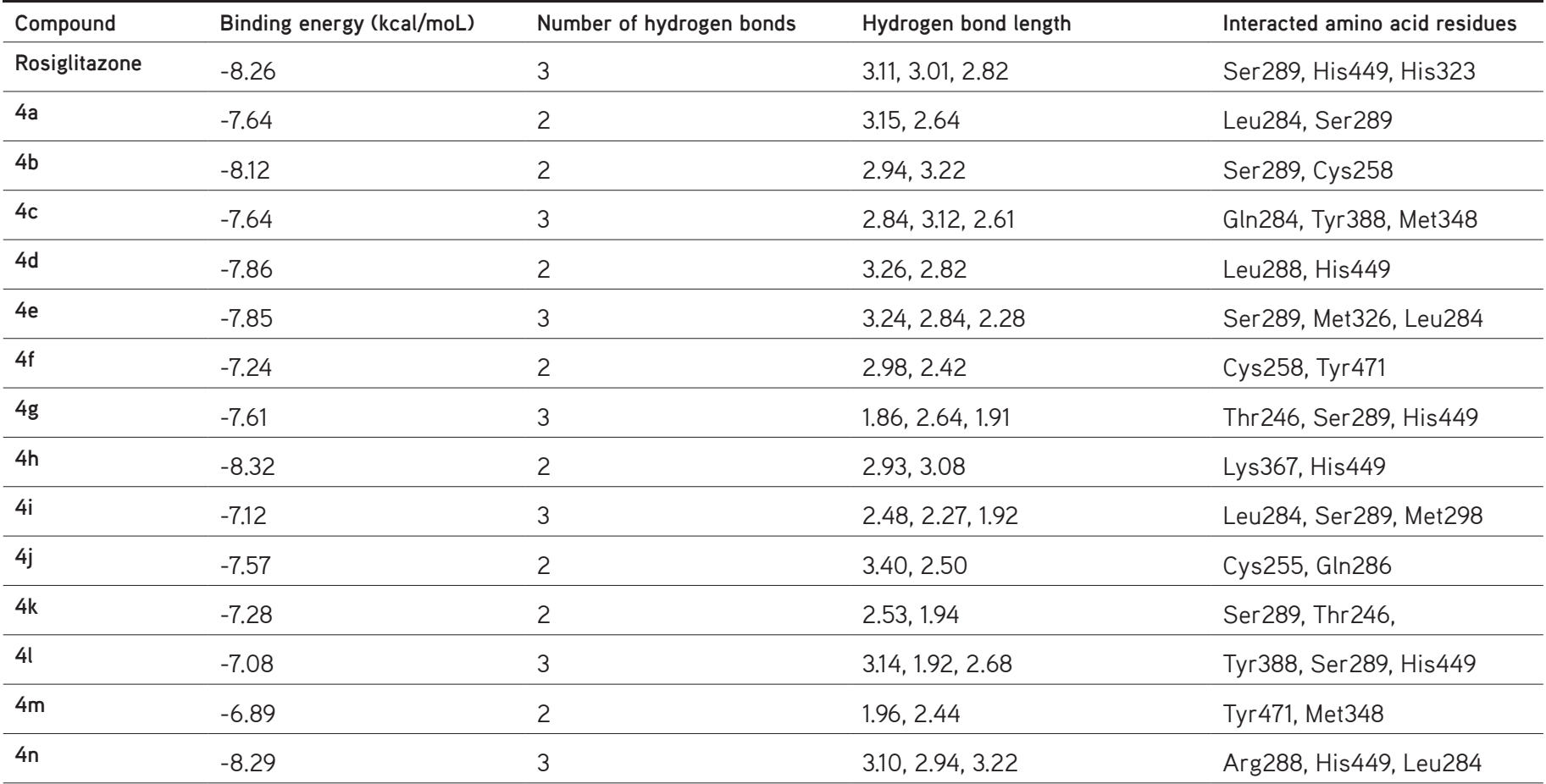

PPAR $\gamma$ : Proliferator-activated receptor gamma

\section{Table 7. Binding energy and amino acid residues interacted by the compounds 4a-4n with the target COX-1 isoenzyme (PDB ID: 1EQG)}

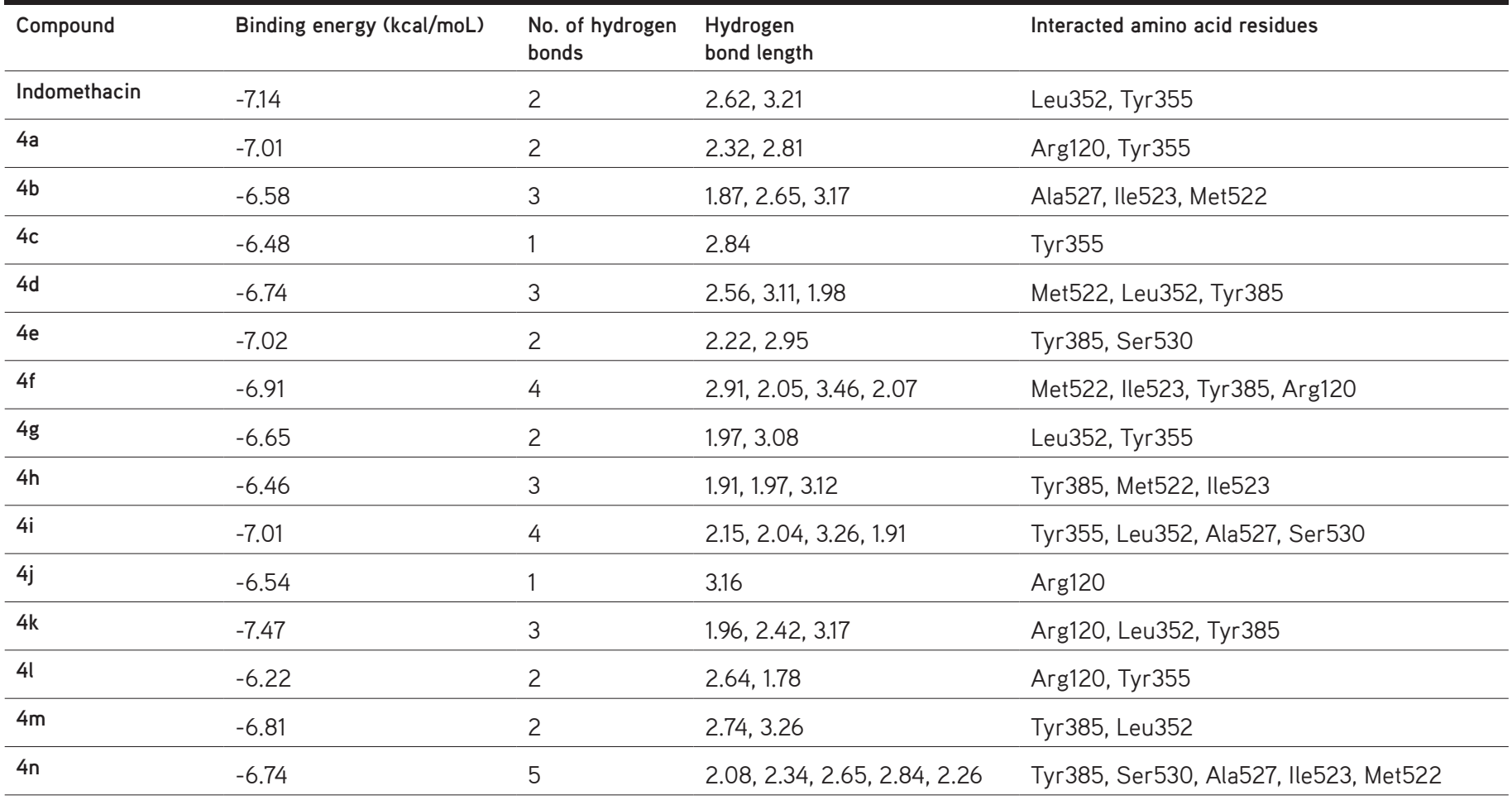


Table 8. Binding energy and amino acid residues interacted by the compounds 4a-4n with the target COX-2 isoenzyme (PDB ID: 1CX2)

\begin{tabular}{|c|c|c|c|c|}
\hline Compound & Binding energy $(\mathrm{kcal} / \mathrm{moL})$ & Number of hydrogen bonds & Hydrogen bond length & Interacted amino acid residues \\
\hline Celecoxib & -10.89 & 5 & $2.25,2.08,2.16,2.84,3.46$ & His90, Gln192, Leu352, Ser353, Phe518 \\
\hline $4 a$ & -8.59 & 3 & $1.95,2.64,3.66$ & Ala527, His90, Ala527 \\
\hline $4 b$ & -7.99 & 3 & $3.22,1.94,2.65$ & Ala527, Ser530, Val523 \\
\hline $4 c$ & -9.47 & 2 & $3.42,2.68$ & Tyr355, VAL349 \\
\hline $4 \mathrm{e}$ & -9.19 & 2 & $1.88,2.86$ & Ser253, Val349 \\
\hline $4 f$ & -9.86 & 3 & $1.94,2.09,2.72$ & His90, Arg120, Ser353 \\
\hline $4 \mathrm{~g}$ & -8.89 & 2 & $2.55,3.74$ & Arg120, Val349 \\
\hline $4 \mathrm{~h}$ & -7.49 & 3 & $2.19,2.92,3.15$ & His90, Ser353, Val523 \\
\hline $4 \mathrm{k}$ & -10.98 & 2 & $2.32,2.87$ & Arg120, Ser353 \\
\hline 41 & -8.33 & 2 & $1.95,2.40$ & Tyr355, Tyr385 \\
\hline $4 m$ & -9.81 & 3 & $3.62,2.31,2.48$ & Arg120, Val349, Ser353 \\
\hline $4 n$ & -7.45 & 2 & $2.85,3.23$ & Arg120, Val349 \\
\hline
\end{tabular}

and morpholine moieties. From the data of in vivo hypoglycemic activity screening, compounds $4 \mathrm{~h}$ and $4 \mathrm{n}$ exhibited good hypoglycemic activity in both acute and chronic toxic studies. In the in vitro anti-inflammatory activity investigation by HRBC membrane stabilization method and protein denaturation method, compound $4 \mathrm{k}$ exhibited good activity among all. From the viewpoint of structure-activity relationship, it was identified that compounds with electron releasing groups at the para position of the phenyl ring may have the ability to produce hypoglycemic activity and the presence of electron withdrawing groups at the para position of the phenyl ring causes antiinflammatory activity. Molecular docking studies with PPAR $\gamma$ receptor protein (PDB ID-2PRG) and compounds $4 \mathrm{~h}$ and $4 \mathrm{n}$ exhibited higher binding affinity. In molecular docking studies with COX isoenzymes (PDB IDs: 1EQG and 1CX2) for potential anti-inflammatory activity, compound $4 \mathrm{k}$ exhibited higher binding affinity.

\section{ACKNOWLEDGEMENTS}

The authors are thankful to the Management of V. V. Institute of Pharmaceutical Sciences, Gudlavalleru-521356, Andhra Pradesh, India, for providing necessary facilities to carry out the research work. The authors are grateful to Dr. K. Ravishankar, Principal, Aditya College of Pharmacy, Surampalem, Andhra Pradesh, for helping with the biological evaluation.

Conflicts of interest: No conflict of interest was declared by the authors.

\section{REFERENCES}

1. Maccari R, Ottanà R, Ciurleo R, Rakowitz D, Matuszczak B, Laggner $\mathrm{C}$, Langer T. Synthesis, induced-fit docking investigations, and in vitro aldose reductase inhibitory activity of non-carboxylic acid containing 2,4-thiazolidinedione derivatives. Bioorg Med Chem. 2008;16:5840-5852.

2. Nazreen S, Alam MS, Hamid H, Yar MS, Dhulap A, Alam P, Pasha MA, Bano S, Alam MM, Haider S, Kharbanda C, Ali Y, Pillai KK. Thiazolidine-2,4-diones derivatives as PPAR- $\gamma$ agonists: synthesis, molecular docking, in vitro and in vivo antidiabetic activity with hepatotoxicity risk evaluation and effect on PPAR- $\gamma$ gene expression. Bioorg Med Chem Lett. 2014;24:3034-3042.

3. Wild S, Roglic G, Green A, Sicree R, King H. Global prevalence of diabetes: estimates for the year 2000 and projections for 2030 . Diabetes Care. 2004:27:1047-1053.

4. Gangadhara S, Prasad Ch, Venkateswarlu P. Synthesis, antimicrobial and antioxidant activities of novel series of cinnamamide derivatives having morpholine moiety. Med Chem. 2014;4:778-783.

5. Vijay P, Kalpana T, Sonali M, Rhea M, Ramaa CS. Synthesis and primary cytotoxicity evaluation of new 5-benzylidene-2,4thiazolidinedione derivatives. Eur J Med Chem. 2010;45:45394544.

6. Lee HW, Kim BY, Ahn JB, Kang SK, Lee JH, Shin JS, Ahn SK, Lee SJ, Yoon SS. Molecular design, synthesis, and hypoglycemic and hypolipidemic activities of novel pyrimidine derivatives having thiazolidinedione. Eur J Med Chem. 2005;40:862-874

7. Nazreen S, Alam MS, Hamid H, Yar MS, Dhulap A, Alam P, Pasha MA, Bano S, Alam MM, Haider S, Kharbanda C, Ali Y, Pillai KK. Thiazolidine-2,4-diones derivatives as PPARy agonists: synthesis, molecular docking, in vitro and in vivo antidiabetic activity with 
hepatotoxicity risk evaluation and effect on PPAR $\gamma$ gene expression. Bioorg Med Chem Lett. 2014;24:3034-3042.

8. Wang Z, Liu Z, Lee W, Kim SN, Yoon G, Cheon SH. Design, synthesis and docking study of 5-(substituted benzylidene) thiazolidine-2,4dione derivatives as inhibitors of protein tyrosine phosphatase 1B. Bioorg Med Chem Lett. 2014;24:3337-3340.

9. Wu $\mathrm{Y}$, Tai $\mathrm{HH}$, Cho $\mathrm{H}$. Synthesis and SAR of thiazolidinedione derivatives as 15-PGDH inhibitors. Bioorg Med Chem. 2010;18:14281433.

10. Lee HW, Kim BY, Ahn JB, Kang SK, Lee JH, Shin JS, Ahn SK, Lee SJ, Yoon SS. Molecular design, synthesis, and hypoglycemic and hypolipidemic activities of novel pyrimidine derivatives having thiazolidinedione. Eur J Med Chem. 2005;40:862-874.

11. Maccari R, Ottanà R, Ciurleo R, Vigorita MG, Rakowitz D, Steindl T, Langer $\mathrm{T}$. Evaluation of in vitro aldose reductase inhibitory activity of 5-arylidene-2,4-thiazolidinediones. Bioorg Med Chem Lett. 2007;17:3886-3893.

12. Youssef AM, White MS, Villanueva EB, El-Ashmawy IM, Klegeris A. Synthesis and biological evaluation of novel pyrazolyl-2,4thiazolidinediones as anti-inflammatory and neuroprotective agents. Bioorg Med Chem. 2010;18:2019-2028.

13. Neeru M, Prasad DN. Synthesis and antimicrobial evaluation of $\mathrm{N}$-substituted-5-benzylidene-2,4-thiazolidinedione derivatives. Iran J Pharm Sci. 2012;8:209-214.

14. Pattan S, Kedar M, Pattan J, Dengale S, Sanap M, Gharate U, Shinde $P$, Kadam $S$. Synthesis and evaluation of some novel 2,4-thiazolidinedione derivatives for antibacterial, antitubercular and antidiabetic activities. Indian J Chem. 2012;51B:1421-1425.

15. Reddy KA, Lohray BB, Bhushan V, Reddy AS, Kishore PH, Rao VV, Saibaba V, Bajji AC, Rajesh BM, Reddy KV, Chakrabarti R, Rajagopalan R. Novel euglycemic and hypolipidemic agents: Part-2. Antioxidant moiety as structural motif. Bioorg Med Chem Lett. 1998;8:999-1002.

16. Hafez HN, El-Gazzar AR. Synthesis and antitumor activity of substituted triazolo [4,3-a] pyrimidin-6-sulfonamide with an incorporated thiazolidinone moiety. Bioorg Med Chem Lett. 2009;19:4143-4147.

17. Fan YH, Chen H, Natarajan A, Guo Y, Harbinski F, lyasere J, Christ W, Aktas H, Halperin JA. Structure-activity requirements for the antiproliferative effect of troglitazone derivatives mediated by depletion of intracellular calcium. Bioorg Med Chem Lett. 2004:14:2547-2550.

18. Mavandadi F, Pilotti A. The impact of microwave-assisted organic synthesis in drug discovery. Drug Discov Today. 2006;11:165-174.

19. Kidwai M. Dry media reactions. Pure Appl Chem. 2001;73:147-151.
20. Kumar BR, Soni M, Kumar SS, Singh K, Patil M, Baig RB, Adhikary L. Synthesis, glucose uptake activity and structure-activity relationships of some novel glitazones incorporated with glycine, aromatic and alicyclic amine moieties via two carbon acyl linker. Eur J Med Chem. 2011;46:835-844.

21. Prashantha Kumar BR, Nanjan MJ, Suresh B, Karvekar MD, Adhikary L. Microwave induced synthesis of the thiazolidine-2,4-dione motif and the efficient solvent free-solid phase parallel syntheses of 5-benzylidene-thiazolidine-2,4-dione and 5-benzylidene-2-thioxothiazolidine-4-one compounds. J Heterocycl Chem. 2006;43:897903.

22. Jiwane SK, Singh VK, Namdeo KP, Prajapati SK. Synthesis of Some Novel 2,4-Thiazolidinedione Derivatives and Their Biological Screening as Antidiabetic Agents. Asian J Chem. 2009;21:50685072.

23. Sivakumar KK, Rajasekaran A, Senthilkumar P, Wattamwar PP. Conventional and microwave assisted synthesis of pyrazolone Mannich bases possessing anti-inflammatory, analgesic, ulcerogenic effect and antimicrobial properties. Bioorg Med Chem Lett. 2014;24:2940-2944.

24. Anna PGN, Dipali D, Hemant DU. Facile synthesis and in vivo hypoglycemic activity of novel 2,4-hiazolidinedione derivatives. Eur J Exp Bio. 2012;2:343-353.

25. Shashikant RP, Prajact K, Ashwini P, Ana N, Kittur BS. Studies on the Synthesis of Novel 2,4-Thiazolidinedione Derivatives with Antidiabetic Activity. Iranian J Pharm Sci. 2009;5:225-230.

26. Sachin SS, Archana RJ, Manoj NG. In vitro antioxidant and antiinflammatory activity of methanol extract of Oxalis corniculata Linn. Int J Pharm Pharm Sci. 2010;2:146-155.

27. Reshma, Arun KP, Brindha P. In vitro anti-inflammatory, Antioxidant and nephroprotective studies on leaves of Aegle marmelos and Ocimum sanctum. Asian J Pharm Clin Res. 2014;7:121-129.

28. Sangita Ch, Priyanka Ch, Protapaditya D, Sanjib B. Evaluation of in vitro anti-inflammatory activity of coffee against the denaturation of protein. Asian Pac J Trop Biomed. 2012;2:S178-S180.

29. Madhuri M, Prasad Ch, Vasudeva Rao A. In silico Protein-Ligand Docking Studies on Thiazolidinediones as Potential Anticancer Agents. Int J Comp Appl. 2014;95:13-16.

30. Arifa B, Shaheen B, Prasad KVSRG, Bharathi K. In silico studies on functionalized azaglycine derivatives containing original article 2,4-thiazolidinedione scaffold on multiple targets. Int J Pharm Pharm Sci. 2017;9:209-215.

31. Ugwu DI, Okoro UC, Ukoha PO, Gupta A, Okafor SN. Novel antiinflammatory and analgesic agents: synthesis, molecular docking and in vivo studies. J Enzyme Inhib Med Chem. 2018;33:405-415. 